\title{
GRK2 suppresses lymphomagenesis by inhibiting the MALT1 proto-oncoprotein
}

\author{
Jing Cheng, ${ }^{1}$ Linda R. Klei, ${ }^{1}$ Nathaniel E. Hubel, ${ }^{1,2}$ Ming Zhang, ${ }^{3}$ Rebekka Schairer, ${ }^{3}$ Lisa M. Maurer, ${ }^{1}$ Hanna B. Klei, ${ }^{1}$ \\ Heejae Kang, ${ }^{2}$ Vincent J. Concel, ${ }^{1}$ Phillip C. Delekta, ${ }^{4}$ Eric V. Dang, ${ }^{5}$ Michelle A. Mintz, ${ }^{5}$ Mathijs Baens, ${ }^{6,7}$ Jason G. Cyster, ${ }^{5,8,9}$ \\ Narayanan Parameswaran, ${ }^{10}$ Margot Thome, ${ }^{3}$ Peter C. Lucas, ${ }^{2}$ and Linda M. McAllister-Lucas ${ }^{1}$
}

\begin{abstract}
1Department of Pediatrics and 2Department of Pathology, University of Pittsburgh School of Medicine and UPMC Hillman Cancer Center, Pittsburgh, Pennsylvania, USA. ${ }^{3}$ Department of Biochemistry, Center of Immunity and Infection, University of Lausanne, Epalinges, Switzerland. ${ }^{4}$ Department of Microbiology and Molecular Genetics, Michigan State University, East Lansing, Michigan, USA. ${ }^{5}$ Department of Biophysics and Biochemistry, UCSF, San Francisco, California, USA. ${ }^{6}$ Human Genome Laboratory, VIB Center for the Biology of Disease, and ${ }^{7}$ Center for Human Cenetics, Katholieke Universiteit Leuven, Leuven, Belgium. ${ }^{8}$ Howard Hughes Medical Institute and ${ }^{9}$ Department of Microbiology and Immunology, UCSF, San Francisco, California, USA. ${ }^{10}$ Department of Physiology, Michigan State University, East Lansing, Michigan, USA.
\end{abstract}

\begin{abstract}
Antigen receptor-dependent (AgR-dependent) stimulation of the NF- $\mathrm{KB}$ transcription factor in lymphocytes is a required event during adaptive immune response, but dysregulated activation of this signaling pathway can lead to lymphoma. AgR stimulation promotes assembly of the CARMA1-BCL10-MALT1 complex, wherein MALT1 acts as (a) a scaffold to recruit components of the canonical NF- $\kappa B$ machinery and (b) a protease to cleave and inactivate specific substrates, including negative regulators of NF-KB. In multiple lymphoma subtypes, malignant B cells hijack AgR signaling pathways to promote their own growth and survival, and inhibiting MALT1 reduces the viability and growth of these tumors. As such, MALT1 has emerged as a potential pharmaceutical target. Here, we identified G protein-coupled receptor kinase 2 (GRK2) as a new MALT1-interacting protein. We demonstrated that GRK2 binds the death domain of MALT1 and inhibits MALT1 scaffolding and proteolytic activities. We found that lower GRK2 levels in activated B cell-type diffuse large B cell lymphoma (ABCDLBCL) are associated with reduced survival, and that GRK2 knockdown enhances ABC-DLBCL tumor growth in vitro and in vivo. Together, our findings suggest that GRK2 can function as a tumor suppressor by inhibiting MALT1 and provide a roadmap for developing new strategies to inhibit MALT1-dependent lymphomagenesis.
\end{abstract}

\section{Introduction}

Diffuse large B cell lymphoma (DLBCL) is the most frequent subtype of non-Hodgkin lymphoma (NHL), accounting for 30\%-40\% of newly diagnosed cases (1). With more than 20,000 deaths from lymphoma occurring each year in the United States (2), new approaches to diagnosis, prognosis, and treatment are needed. Although the molecular and genetic features that drive aggressive behavior of B cell lymphomas are not fully defined, gene expression profiling studies have identified at least 2 distinct molecular subtypes of DLBCL, termed germinal center B cell (GCB) and activated $B$ cell (ABC) (3). ABC-DLBCL exhibits an inferior outcome following standard R-CHOP chemotherapy, with a 3-year progression-free survival of approximately $40 \%$ compared with $75 \%$ for non- $\mathrm{ABC}$ cases, and is associated with constitutive canonical NF- $\kappa \mathrm{B}$ activation $(4,5)$. A loss-of-function RNA interference screen revealed that the majority of ABC-DLBCL cell lines rely on components of the $\mathrm{B}$ cell receptor-dependent (BCR-dependent) $\mathrm{NF}-\kappa \mathrm{B}$ signaling pathway for growth and survival (6).

Authorship note: PCL and LMML contributed equally as senior authors Conflict of interest: The authors have declared that no conflict of interest exists. Copyright: (5) 2020, American Society for Clinical Investigation.

Submitted: August 28, 2017; Accepted: November 6, 2019; Published: January 21, 2020. Reference information: / Clin Invest. 2020;130(2):1036-1051.

https://doi.org/10.1172/JCI97040.
Stimulation of the B or T cell antigen receptor (AgR) results in assembly and activation of the CBM complex, which is composed of the scaffolding protein CARMA1 (also known as CARD11), the adaptor protein BCL10, and the protease MALT1 $(7,8)$. Loss of any component of this complex is toxic for ABC-DLBCL cells, indicating that it plays a critical role in the molecular pathogenesis of this tumor (6). MALT1 functions as the essential downstream effector molecule of the CBM complex by carrying out 2 important functions. First, MALT1 acts as a scaffold to recruit downstream signaling proteins, most notably the ubiquitin ligase TNF receptor-associated factor 6 (TRAF6). In turn, TRAF6 directs an array of polyubiquitinations that promote stimulation of the I $\kappa$ B kinase (IKK) complex (9-11). IKK then phosphorylates the inhibitor of $\mathrm{NF}-\kappa \mathrm{B}(\mathrm{I} \kappa \mathrm{B})$, thereby targeting it for proteasomal degradation and freeing NF- $\kappa \mathrm{B}$ subunits to translocate into the nucleus and alter target gene expression (12). Second, MALT1 acts as protease to enzymatically cleave and inactivate multiple substrates, including several negative regulators of canonical NF- $\mathrm{BB}$ signaling (13, 14). Thus, MALT1 protease activity is thought to amplify and sustain NF- $\kappa \mathrm{B}$ activation by clearing proteins that dampen this pathway. For example, activated MALT1 protease cleaves the NF- $\mathrm{BB}$ family member RELB (15). Since RELB is an inhibitor of canonical RELA-dependent transcriptional activity, MALT1-dependent cleavage of RELB results in enhanced expression of canonical NF- $\kappa B$ gene targets. MALT1 also cleaves several other substrates, 
including its binding partner BCL10 and the deubiquitinases A2O and cylindromatosis (CYLD) (15-19). Collectively, these cleavage events not only optimize NF- $\kappa$ B activation but also regulate cellular adhesion and enhance the related c-Jun N-terminal kinase (JNK) signaling pathway (20).

In ABC-DLBCL, mutations in the CD79A or CD79B subunits of BCR are present in approximately $23 \%$ of cases $(21,22)$, and gain-of-function mutations of CARMA1 occur in another $9 \%$ of cases $(1,22-24)$. These mutations mimic BCR signaling and consequently result in constitutive MALT1-mediated NF- $\kappa \mathrm{B}$ activation. Blockade of MALT1 activity, by either genetic knockdown or chemical inhibition of protease activity, reduces viability and growth of ABC-DLBCL lines (25-29). In addition to ABC-DLBCL, inappropriate MALT1 activation also occurs in a variety of other NHL subtypes and is critical to tumor pathogenesis. Translocations $t(1 ; 14)$ and $t(14 ; 18)$ in MALT lymphoma position BCL10 and MALT1, respectively, adjacent to the Ig heavy chain enhancer, leading to BCL10 and MALT1 overexpression and NF- $\kappa$ B activation (12). The $\mathrm{t}(11 ; 18)$ translocation, which occurs in $30 \%$ of MALT lymphomas, creates an oncogenic fusion protein that links the N-terminus of cellular inhibitor of apoptosis (API2) to the MALT1 C-terminus, and this results in constitutive MALT1 proteolytic activity (30-32). Furthermore, recent studies have suggested that a subset of mantle cell lymphomas, peripheral $\mathrm{T}$ cell lymphomas, and $\mathrm{T}$ cell leukemias may also require MALT1 proteolytic activity for survival (33-35). In light of these observations, MALT1 has emerged as a promising new target for pharmaceutical intervention in the treatment of multiple subtypes of lymphoid malignancy.

Here, we identify G protein-coupled receptor kinase 2 (GRK2) as a new MALT1-interacting protein and provide compelling evidence that GRK2 inhibits MALT1-mediated NF- $\kappa$ B activity in lymphoma cells, which leads to reduced tumor growth. GRK2 is 1 of 7 members of the GRK family, a group of proteins best known as serine/threonine kinases that phosphorylate activated $G$ proteincoupled receptors, signaling receptor desensitization (36). Emerging evidence indicates that in addition to this canonical function, GRK2 binds to a variety of other cellular proteins involved in diverse aspects of signal transduction, and regulates the activity of these signaling proteins via kinase-independent mechanisms (37). While GRK2 has been reported to influence many of the processes involved in the hallmarks of cancer, such as cell proliferation, survival, motility metabolism, and others, the role of GRK2 in tumor formation and progression has only recently begun to be investigated (38). GRK2 is expressed in several cell types within the immune system, though relatively little is known about the function of GRK2 in either normal or malignant lymphocytes (39). Our findings suggest that GRK2 can act as a tumor suppressor by binding and inhibiting MALT1. This study provides important new insight into the molecular mechanisms that regulate the MALT1 proto-oncoprotein and represents a major step toward developing improved prognostication and targeted therapeutics for MALT1-dependent lymphomas.

\section{Results}

GRK2 binds to MALT1 in both T cells and B cells and dissociates from MALT1 after AgR stimulation. Using coimmunoprecipitation (co-IP) coupled with liquid chromatography-mass spectrometry, we identified GRK2 as a new MALT1-interacting protein (Supplemental Figure 1A; supplemental material available online with this article; https://doi.org/10.1172/JCI97040DS1). We validated this interaction by Western blot analysis, showing endogenous GRK2 and MALT1 co-IP in both Jurkat T cells and BJAB B cells (Figure 1A), as well as in HEK293T cells (Supplemental Figure 1B). We then used purified recombinant proteins to demonstrate a direct interaction between GRK2 and MALT1, but not between GRK2 and BCL10, another member of the CBM complex (Figure $1 \mathrm{~B}$ and Supplemental Figure 1C). Next, we investigated whether AgR stimulation, which promotes both MALT1 scaffolding and proteolytic activities, impacts the GRK2-MALT1 interaction. We found that treatment with phorbol ester 12-myristate 13-acetate and the calcium ionophore ionomycin (PMA/Iono), which mimics AgR stimulation by promoting PKC-dependent phosphorylation of CARMA1 and subsequent activation of the CBM complex (40, 41), resulted in time-dependent dissociation of GRK2/MALT1 in Jurkat T cells (Figure 1, C and D). Notably, GRK2 dissociation from MALT1 occurred within the same general time course as CARMA1 association with BCL10/MALT1 and phosphorylation of IкB. Similar results were also observed in BJAB B cells (Supplemental Figure 1D). These findings could suggest that GRK2 binding to MALT1 inhibits CBM complex function, with dissociation of GRK2 somehow allowing full activation of MALT1 and downstream NF-кB pathway signaling following lymphocyte AgR stimulation.

The GRK2 N-terminus interacts with MALT1 death domain and inhibits MALT1-dependent NF- $\kappa B$ activation. As an initial approach to testing whether GRK2 binding to MALT1 plays an inhibitory role in lymphocyte signaling, we investigated the impact of overexpression of GRK2. We found that GRK2 overexpression inhibited the coimmunoprecipitation of BCL10 and MALT1 (Supplemental Figure 2A). In addition, using differentially epitope-tagged MALT1 monomers, we demonstrated that GRK2 overexpression inhibited BCL10-dependent MALT1 oligomerization (Supplemental Figure 2B). While these overexpression studies suggest that GRK2 might interfere with the BCL10-MALT1 interaction, we did not observe an increase in the association of BCL10 and MALT1 upon GRK2 dissociation after PMA/Iono treatment in lymphocytes (Figure 1C).

We next sought to identify the specific region of MALT1 that interacts with GRK2. MALT1 contains 2 Ig-like protein-protein interaction domains that are required for binding to BCL10 (refs. 42-44 and Figure 2A), and a central catalytic domain that shares homology with the proteolytic active site of the caspase family of serine proteases (43). MALT1 also contains an N-terminal death domain (DD), and recent studies suggest that this domain may interact with BCL10 (44). Interestingly, co-IP mapping studies revealed that GRK2 binds to this MALT1 DD (amino acids 1-139) (Figure 2A). Notably, a recent report demonstrated that MALT1 undergoes autoproteolysis in response to $\mathrm{AgR}$ stimulation, cleaving itself after arginine-149 (R149), thereby separating this DD from the remaining 76-kDa (p76) C-terminal portion of MALT1 (45). In contrast to full-length (FL) MALT1, the p76 cleavage fragment potently activates NF- $\kappa$ B independently of CARMA1/BCL10. These findings suggest that the DD may possess a negative regulatory function and serve to somehow restrain MALT1-dependent signaling.

Together, our findings that GRK2 dissociates from MALT1 in response to AgR stimulation and that GRK2 binds to the MALT1 
A

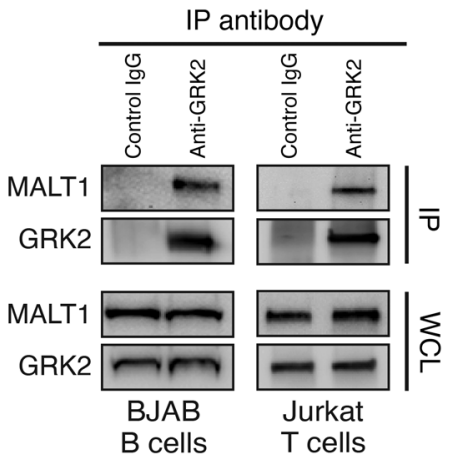

B

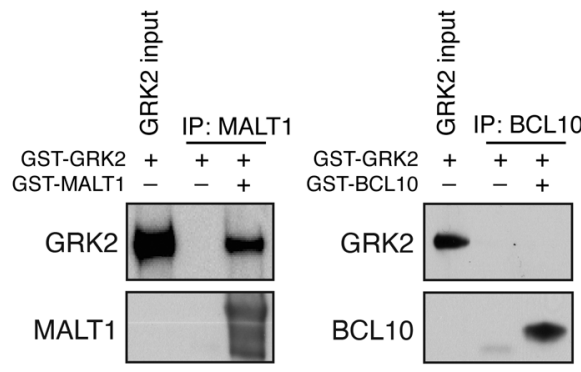

D

C
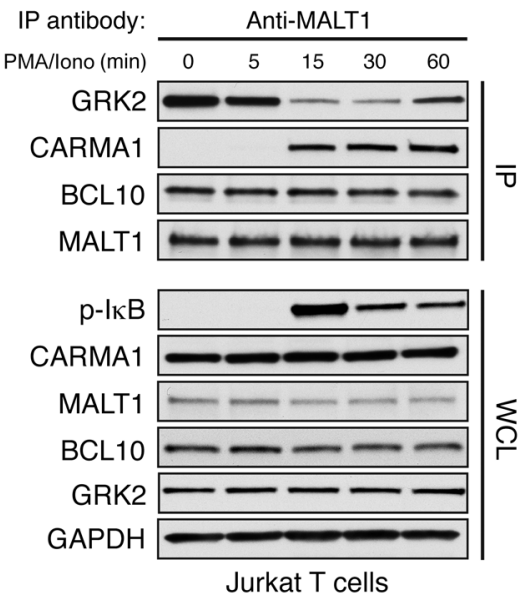

Figure 1. GRK2 binds to MALT1 and dissociates from MALT1 after AgR stimulation. (A) Endogenous MALT1 and GRK2 interact in BJAB $B$ and Jurkat T cells. Coimmunoprecipitation (co-IP) of MALT1 with GRK2 was demonstrated by Western blot. (B) Co-IP analysis reveals that purified recombinant GRK2 interacts directly with MALT1 (left) but not BCL10 (right). (C) AgR stimulation leads to GRK2/MALT1 dissociation in the same general time course as CARMA1 association with BCL10/MALT1. Jurkat T cells were serum-starved and exposed to PMA (50 $\mathrm{ng} / \mathrm{mL}$ )/ionomycin ( $1 \mu \mathrm{M})$ (PMA/lono) for the indicated times. Cell lysates were subjected to immunoprecipitation (IP) with anti-MALT1, followed by immunoblotting with either anti-GRK2, anti-CARMA1, or anti-BCL10. (D) Reverse immunoprecipitation with anti-CRK2 also confirmed that AgR stimulation leads to GRK2/MALT1 dissociation in Jurkat T cells. Cells were treated as in C, and cell lysates were subjected to immunoprecipitation with anti-GRK2, followed by immunoblotting with anti-MALT1. All blots shown are representative of 3 separate experiments.
DD could suggest that GRK2 exerts an inhibitory effect on MALT1dependent signaling, which is relieved after AgR stimulation. Indeed, we found that GRK2 inhibited BCL10/MALT1-dependent $\mathrm{NF}-\kappa \mathrm{B}$ activation (Figure 2B, left). Notably, the kinase-deficient K220R GRK2 mutant (GRK2 K220R) (46) was equally as effective as wild-type (WT) GRK2 at inhibiting BCL10/MALT1-dependent NF- $\kappa \mathrm{B}$ activation, indicating that GRK2 kinase activity is not required for this effect. Importantly, GRK2 did not inhibit NF- $\mathrm{B}$ signaling triggered by the API2-MALT1 fusion oncoprotein (Figure 2B, middle) or by the p76 MALT1 C-terminal autoproteolytic cleavage fragment (Figure 2B, right), both of which are constitutively active forms of MALT1 that lack the DD. These results are consistent with the notion that GRK2-dependent inhibition of MALT1 signaling requires the presence of the MALT1 DD.

Given the strong indications that interaction with GRK2 negatively impacts MALT1 activity, we sought to more precisely characterize how GRK2 interfaces with MALT1. As a first step, we identified the specific region within GRK2 that is responsible for MALT1 binding. Our analysis revealed that the site of MALT1 interaction is located within the N-terminal amino acids (aa 1-173) of GRK2 (Figure 2C). This GRK2 region is composed of the extreme N-terminal helix (referred to as $\alpha \mathrm{N}$ ) (aa 1-20) and the regulator of $\mathrm{G}$ protein signaling homology $(\mathrm{RH})$ protein-protein interaction domain (aa 30-173). Notably, this GRK2 fragment (aa 1-173) alone inhibited BCL10/MALT1-dependent NF-אB activation in a concentration-dependent manner (Figure 2D) and was as effective as full-length GRK2 at blocking BCL10/MALT1 signaling (Supplemental Figure 2C). Similarly to full-length GRK2, expres- sion of this GRK2(1-173) fragment also effectively inhibited the coimmunoprecipitation of BCL10 and MALT1 (Supplemental Figure 2D). Our results indicate that the other domains within GRK2, such as the kinase and pleckstrin homology $(\mathrm{PH})$ domains, are not required for MALT1 inhibition.

GRK2 inhibits MALT1 proteolytic activity. In order to investigate whether GRK2 modulates MALT1 catalytic activity, we first analyzed whether expression of GRK2 in HEK293T cells impacts the proteolytic processing of CYLD or RELB, 2 known MALT1 substrates. We found that BCL10/MALT1-dependent cleavage of CYLD and RELB were both inhibited by expression of GRK2, while API2-MALT1-mediated cleavage of both substrates was not affected (Figure 3, A and B). This lack of effect on API2-MALT1 proteolytic activity is presumably due to the fact that the API2-MALT1 fusion does not retain the DD of MALT1 (31), and parallels the finding noted above that GRK2 does not block API2-MALT1-dependent NF- $\kappa \mathrm{B}$ activation (Figure $2 \mathrm{~B})$. We also performed fluorescence resonance energy transfer (FRET) analysis, which demonstrated that both full-length GRK2 and the GRK2 $\alpha \mathrm{N} / \mathrm{RH}$ fragment (aa 1-173) inhibited BCL10/MALT1-mediated cleavage of the YFP-LVSRCFP fluorescent MALT1 substrate in a concentration-dependent fashion (Figure 3C). This parallels our finding that the GRK2 $\alpha \mathrm{N} /$ RH fragment (aa 1-173) is as effective as full-length GRK2 in blocking BCL10/MALT1-dependent NF- $\mathrm{BB}$ luciferase activation.

To complement our cell-based analyses, we also analyzed the cleavage of the LVSR-AMC fluorogenic MALT1 peptide substrate in a cell-free system. This peptide is based on the MALT1 cleavage site within RELB and is the most efficiently cleaved MALT1 pep- 
A

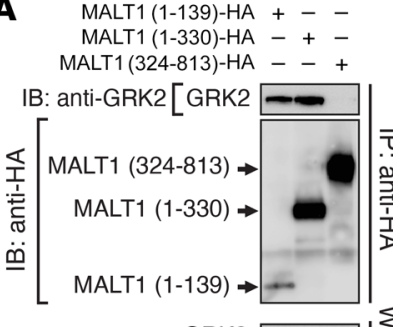

GRK2 --

B

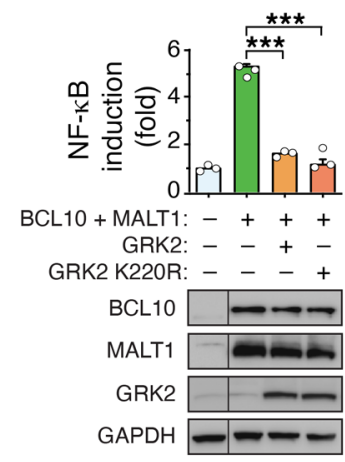

C

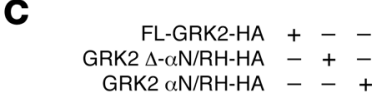

IB: anti-MALT1 [MALT1 - -

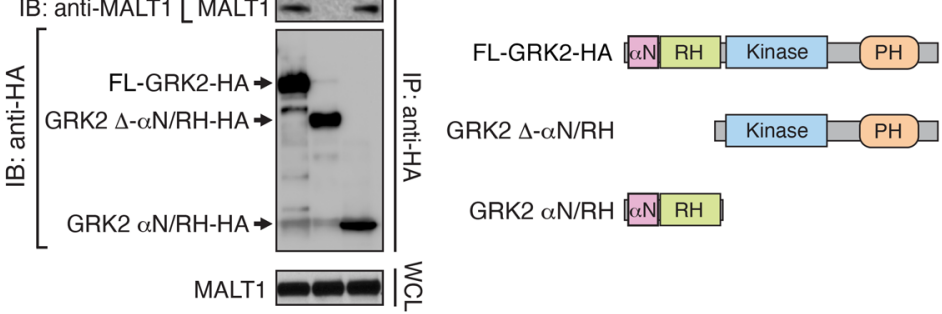

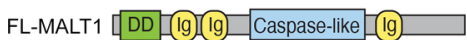
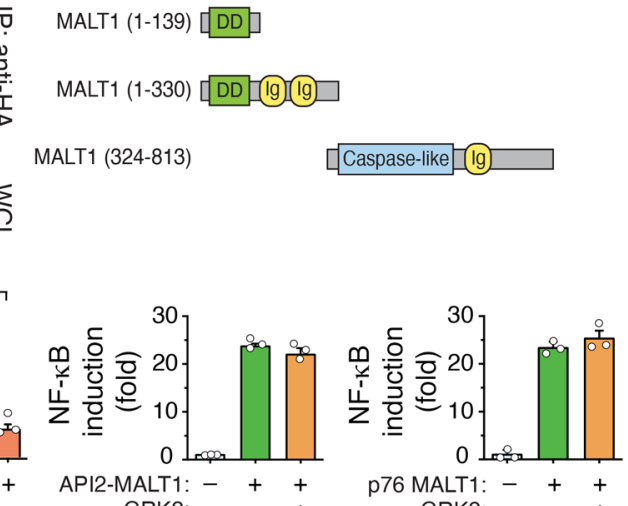

GRK2: - - +

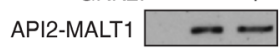

GRK2

GAPDH

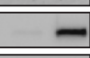

P76 MALT1: -++

GRK2: - - +

p76 MALT1 -

GRK2

GAPDH $-\ldots$
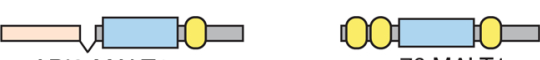

p76 MALT1
Figure 2. GRIK2 N-terminus binds to MALT1 death domain and inhibits MALT1-dependent NF-KB activation. (A) GRK2 binds to the MALT1 death domain (DD). Co-IP of HA-tagged MALT1 constructs with endogenous GRK2 in HEK293T cells is shown (left). Blot is representative of 3 independent experiments. Schematic of domain structures of full-length (FL) MALT1 and deletion mutants is shown at right. (B) Both WT and kinase-deficient (K220R) GRK2 inhibit BCL10/MALT1-induced $\mathrm{NF}-\mathrm{\kappa B}$ luciferase reporter activity (left). Lanes were run on the same gel and were noncontiguous in the leftmost blot; the vertical dividing line shows where images are spliced together. GRK2 does not affect API2-MALT1-induced (middle) or p76 MALT1-induced (right) NF- $\kappa B$ luciferase reporter activity ( $n$ = 3). (C) CRK2 $\alpha$ N/RH (aa 1-173) interacts with endogenous MALT1. Proteins were expressed in HEK293T cells, and co-IP was assessed by Western blot (left). Blot is representative of 3 independent experiments. Domain structures of full-length GRK2 and deletion mutants are shown at right. (D) The GRK2 $\alpha$ N/RH fragment (aa 1-173) inhibits BCL10/MALT1-induced NF- $\mathrm{KB}$ luciferase reporter activity in a dose-dependent manner $(n=3)$. All values are represented as mean \pm SEM. ${ }^{* *} P<0.01$, ${ }^{* * *} P<0.001$, by 1 -way ANOVA, followed by Tukey's multiplecomparisons test.

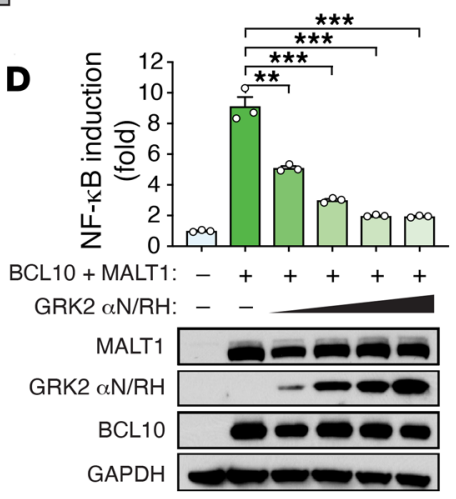

tide substrate currently available (15). Using purified recombinant human proteins, we found that addition of GRK2 into the reaction reduced the cleavage of LVSR-AMC by MALT1 (Figure 3D). This analysis with recombinant purified MALT1 demonstrates that BCL10 does not need to be present for GRK2 to exert an inhibitory effect on MALT1 protease activity. Together, our data in both cell-based and cell-free systems provide convincing evidence that GRK2 inhibits MALT1 proteolytic activity.

GRK2 attenuates MALT1 scaffolding and proteolytic activities, $N F-\kappa B$ induction, and IL-2 production in stimulated $T$ cells. To begin evaluating the impact of GRK2 on MALT1 activity in lymphocytes, we transiently transfected Jurkat T cells with either WT or kinase-deficient (K22OR) GRK2 and then stimulated the cells with PMA/Iono. We found that increased expression of either WT GRK2 or K220R kinase-dead GRK2 mutant abrogated PMA/Ionoinduced NF- $\kappa \mathrm{B}$ activation (Figure $4 \mathrm{~A}$, top). In contrast, GRK2 had no effect on NF- $\kappa$ B induction by TNF, which is known to occur via a signaling pathway that is independent of MALT1 (Figure 4A, bottom, and ref. 47). To complement this analysis, we performed the converse experiment and stably knocked down GRK2 in Jurkat T cells using lentiviral shRNA. In order to rule out off-target effects of the shRNA, we used 3 distinct shRNAs targeting different regions of GRK2 (designated as shRNAs 1, 2, and 3). First, we examined the impact of GRK2 depletion on AgR-induced CBM complex formation. While we did not observe a significant change in the interaction between MALT1 and BCL10, PMA/Iono-induced co-IP of CARMA1 with MALT1 was significantly enhanced after stable GRK2 knockdown, suggesting that GRK2 may inhibit overall CBM complex assembly (Figure 4B and Supplemental Figure $3 A)$. Second, we also examined AgR-induced IкB phosphorylation, a signaling event that is dependent on MALT1 scaffolding activity but does not require MALT1 proteolytic activity $(15,17$, 20). Strikingly, although our shRNA-mediated GRK2 knockdown was incomplete, cells responded to PMA/Iono or anti-CD3/CD28 with substantially more robust I $\mathrm{B}$ phosphorylation when GRK2 levels were reduced (Figure 4B and Supplemental Figure 3, A and B). Consistent with our observation that enforced expression of GRK2 has no effect on TNF- $\alpha$-dependent NF- $\kappa$ B transcriptional activation, we found that TNF- $\alpha$-induced I $\mathrm{B}$ phosphorylation remained unaffected after GRK2 knockdown. Third, we evaluated the effect of GRK2 knockdown on MALT1 proteolytic activity by comparing the ratios of cleaved CYLD and RELB fragment levels to full-length protein levels in control and GRK2-depleted $\mathrm{T}$ cells. We found that the relative levels of cleaved CYLD (Figure 4C) and cleaved RELB (Figure 4D and Supplemental Figure 3C) in response to PMA/Iono or $\mathrm{CD} 3 / \mathrm{CD} 28$ stimulation in $\mathrm{T}$ cells were 
A

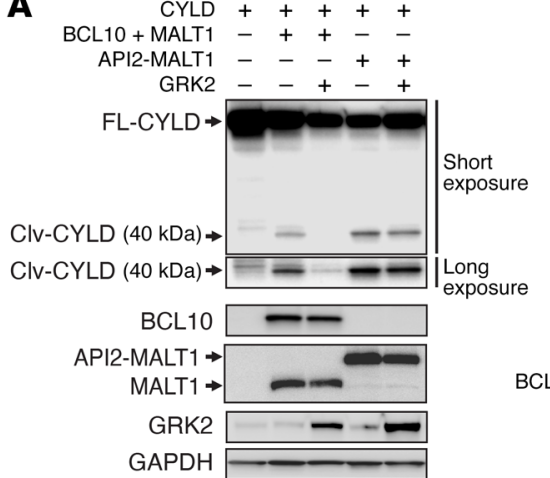

B
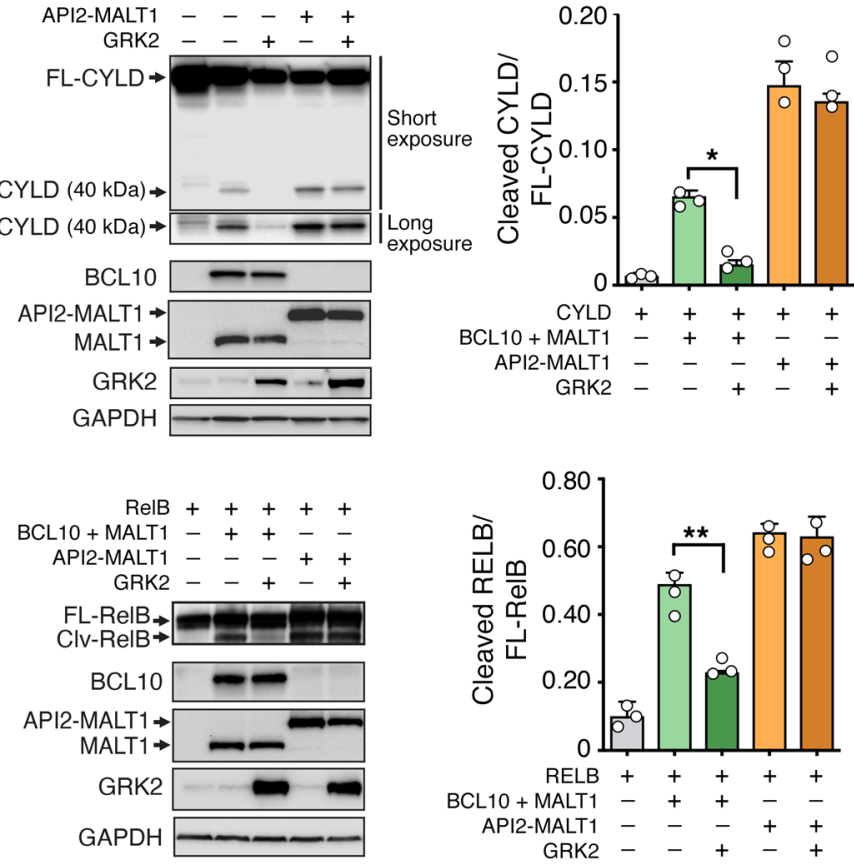

GRK2 $-\quad-\quad+\quad+\quad+$
C
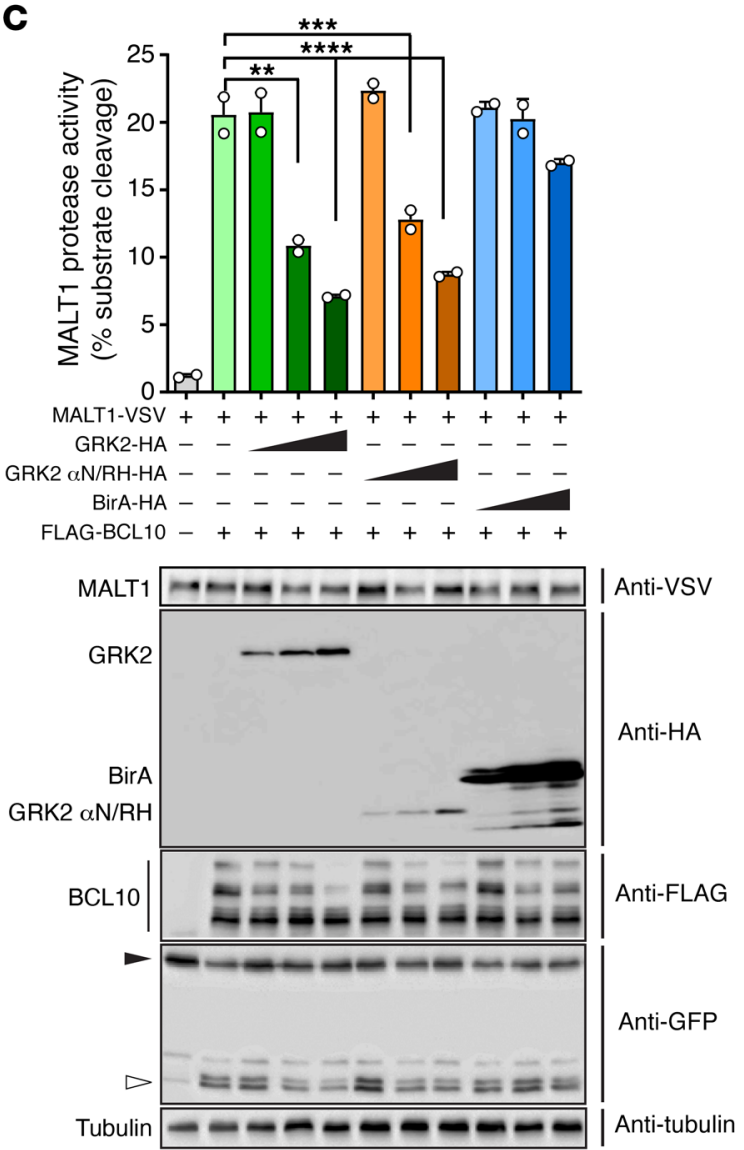

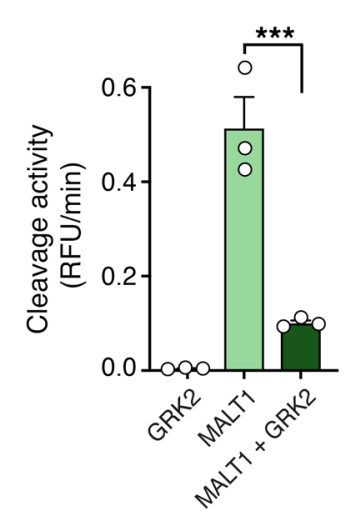

D

Figure 3. GRK2 inhibits MALT1 proteolytic activity. (A and B) GRK2 inhibits MALT1-mediated cleavage of CYLD and RELB. Recombinant proteins were expressed in HEK293T cells, and cleavage of CYLD (A) or RELB (B) was assessed by Western blot. Quantification of the cleavage is shown to the right of the blots. Densitometric analysis was performed using AlphaView software (ProteinSimple) $(n=3)$. (C) Both GRK2 and GRK2 $\alpha$ N/RH (aa 1-173) inhibit MALT1 protease activity in a dose-dependent manner. Indicated proteins were expressed in HEK293T cells together with the eYFP-Leu-Val-Arg-eCFP reporter construct. Cells were evaluated using flow cytometry 24 hours after transfection. Filled and open arrowheads indicate full-length and cleaved reporter, respectively, detected by GFP antibody $(n=2)$. (D) GRK2 inhibits in vitro MALT1 cleavage of the LVSR-AMC substrate in a cell-free system. Recombinant purified proteins were incubated with $50 \mu \mathrm{M}$ Ac-LVSR-AMC. Cleavage activity of MALT1 was determined by the increase of AMC fluorescence measured with a Synergy microplate reader $(n=3)$. All values are represented as mean \pm SEM. ${ }^{*} P<0.05,{ }^{* *} P<0.01,{ }^{* *} P<0.001,{ }^{* * * *} P<0.0001$, by 1 -way ANOVA, followed by Tukey's multiple-comparisons test.

significantly higher after GRK2 shRNA knockdown. In addition, we observed enhanced p65 nuclear translocation (Supplemental Figure 3D) and DNA binding (Supplemental Figure 3E) when GRK2 was stably knocked down. Together, our results indicate that GRK2 depletion in T lymphocytes results in enhanced MALT1 scaffolding and proteolytic activities, leading to enhanced activation of canonical NF- $\kappa \mathrm{B}$ signaling.

Next, to determine the downstream impact of GRK2-mediated MALT1 inhibition on T cell activation, we used ELISA to compare the level of secreted IL-2 after PMA/Iono or CD3/CD28 stimulation in control and GRK2-deficient Jurkat T cells. We chose to focus on IL-2 because it is an NF-kB-inducible cytokine produced in activated $\mathrm{T}$ cells after AgR stimulation that plays a pivotal role in the $\mathrm{T}$ cell immune response (48). We found that GRK2 shRNA knockdown resulted in significantly increased IL-2 secretion (Figure $4 \mathrm{E}$ and Supplemental Figure 3F).

Moreover, we also analyzed the impact of GRK2 deficiency using primary B cells isolated from the spleens of B cell-specific GRK2-knockout mice (Mb1-cre ${ }^{+} G R K 2^{\mathrm{I} / \mathrm{I}}$ ) (49). We found that $\mathrm{PMA} /$ Iono-induced IкB phosphorylation was also enhanced in GRK2-deficient primary B cells when compared with control primary B cells (Mb1-cre-GRK2 ${ }^{\mathrm{Al} /+}$ ) (Supplemental Figure 3G).

To even further confirm the influence of GRK2 on MALT1 activity, we performed targeted deletion of GRK2 in Jurkat $\mathrm{T}$ 

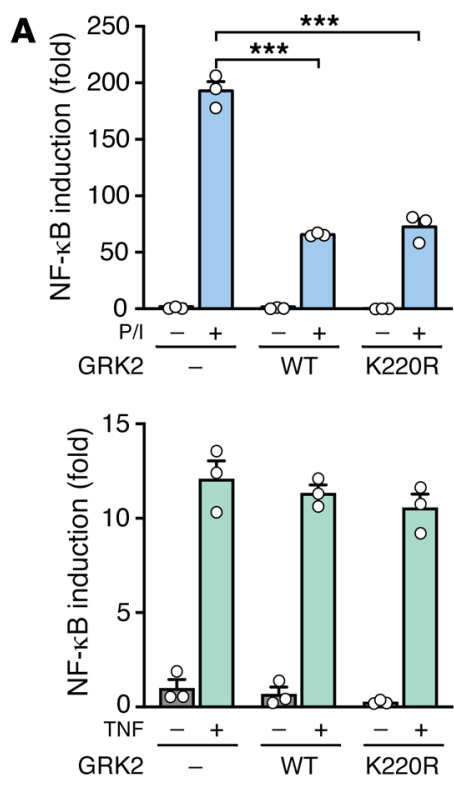

B
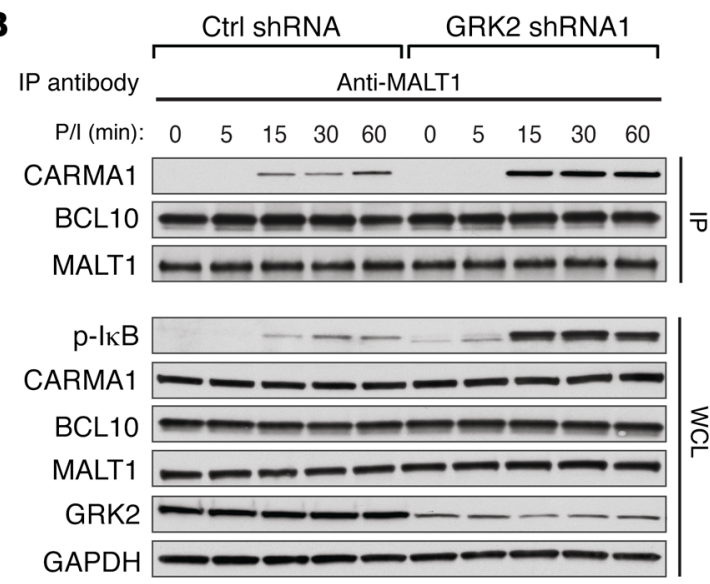

C
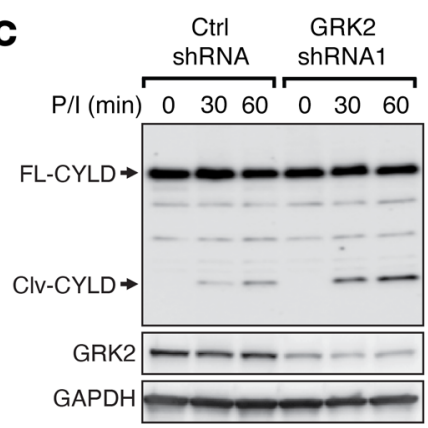

$\sum_{\check{c}}$

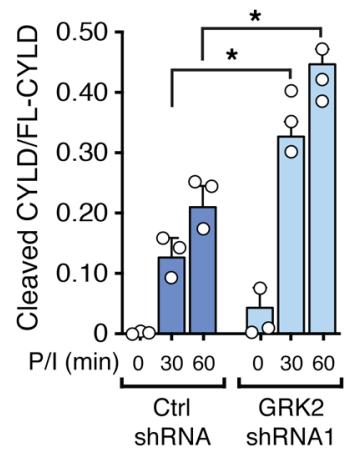

D
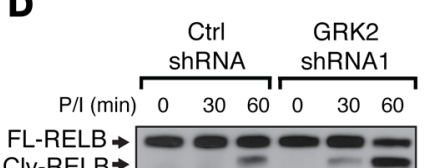

Clv-RELB $\rightarrow$

GRK2

GAPDH $\mathrm{CD} 3 / \mathrm{CD} 28$ (min) $0 \quad 30 \quad 60 \quad 0 \quad 30 \quad 60$

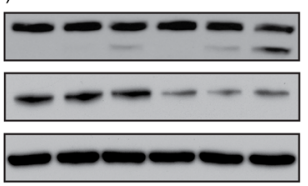

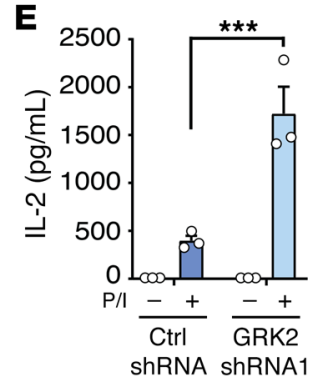

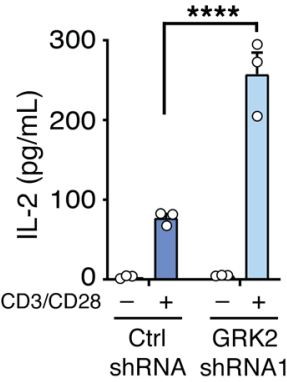

Figure 4. GRK2 attenuates AgR stimulation-induced NF-KB activation, CBM complex formation, MALT1 activity, and IL-2 production in Jurkat T cells. (A) Expression of WT GRK2 or kinase-deficient (K220R) GRK2 mutant in Jurkat T cells inhibits PMA/ionomycin-induced (P/I-induced) (top) but not TNFinduced (bottom) NF-KB luciferase reporter activity $(n=3)$. (B) Knockdown of GRK2 in Jurkat T cells leads to enhanced P/I-induced CBM complex formation. Jurkat T cells were subjected to knockdown with either control or GRK2 shRNA lentivirus (GRK2 shRNA1) and then treated with or without P/l. Binding of BCL10 and CARMA1 to immunoprecipitated MALT1 and phosphorylation of IKB were examined. Blot is representative of 2 independent experiments. (C) GRK2 knockdown in Jurkat T cells leads to increased cleavage of CYLD in response to P/I stimulation. Quantification of the cleavage is shown below. Densitometric analysis was performed using AlphaView software $(n=3)$. (D) GRK2 knockdown in Jurkat T cells leads to increased cleavage of RELB in response to P/I (left) or anti-CD3/CD28 (right) stimulation. Blots are representative of 3 independent experiments. (E) GRK2 knockdown leads to enhanced IL-2 production in Jurkat T cells. Cells were treated with or without P/I (left) or anti-CD3/CD28 (right) for 24 hours, and IL-2 in supernatant was measured by ELISA $(n=3)$. All values are represented as mean \pm SEM. ${ }^{*} P<0.05$, ${ }^{* *} P<0.001,{ }^{* * * *} P<0.0001$. Data from A, C, and E were analyzed by 2 -way ANOVA, followed by Tukey's multiple-comparisons test.

cells using CRISPR/Cas9. Guide RNA (gRNA) with the highest cleavage efficiency was selected for use in the generation of the knockout cell lines (Supplemental Figure 4A). After selecting and expanding individual clones (designated as Jurkat T3, T14, and T26), we confirmed the targeted disruption of GRK2 using Sanger sequencing. TIDE (Tracking Indels by DEcompositon) analysis (50) was used to identify insertion or deletion (INDEL) on individual alleles (Supplemental Figure 4B). We also performed Western blot and confirmed that no detectable GRK2 was seen in the Jurkat GRK2-knockout clones (Figure 5A and Supplemental Figure 4, C and D). As expected based on our shRNA knockdown analysis, the GRK2-CRISPR-knockout Jurkat clones demonstrated enhanced IKB phosphorylation, RELB cleavage, and IL-2 production in response to PMA/Iono or CD3/CD28 stimulation (Figure 5, A-C, and Supplemental Figure 4, C-E). Next, we rescued the homozygous GRK2-CRISPR-knockout Jurkat clone (T3) using lentivirus expressing WT GRK2. Western blot showed that the level of rescued GRK2 expression was comparable to the endogenous level of GRK2 in control Jurkat cells (Supplemental Figure 4F). We found that GRK2 rescue reversed the enhanced IאB phosphorylation, RELB cleavage, and IL-2 production caused by GRK2 knockout (Figure 5, D-F). Taken together, our results convincingly demonstrate that GRK2 inhibits both MALT1 scaffolding and proteolytic activity, thereby abrogating AgR-induced CBM-dependent NF- $\mathrm{\kappa B}$ activation in Jurkat $\mathrm{T}$ cells.

Lower GRK2 expression level is associated with reduced survival in $A B C-D L B C L$. Since our data indicate that GRK2 binds and inhibits MALT1, we wondered whether GRK2 might play an important role 
GRK2 rescue (Jurkat clone T3)

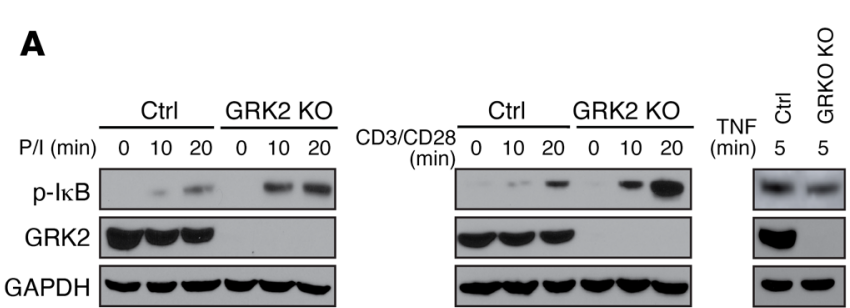

B
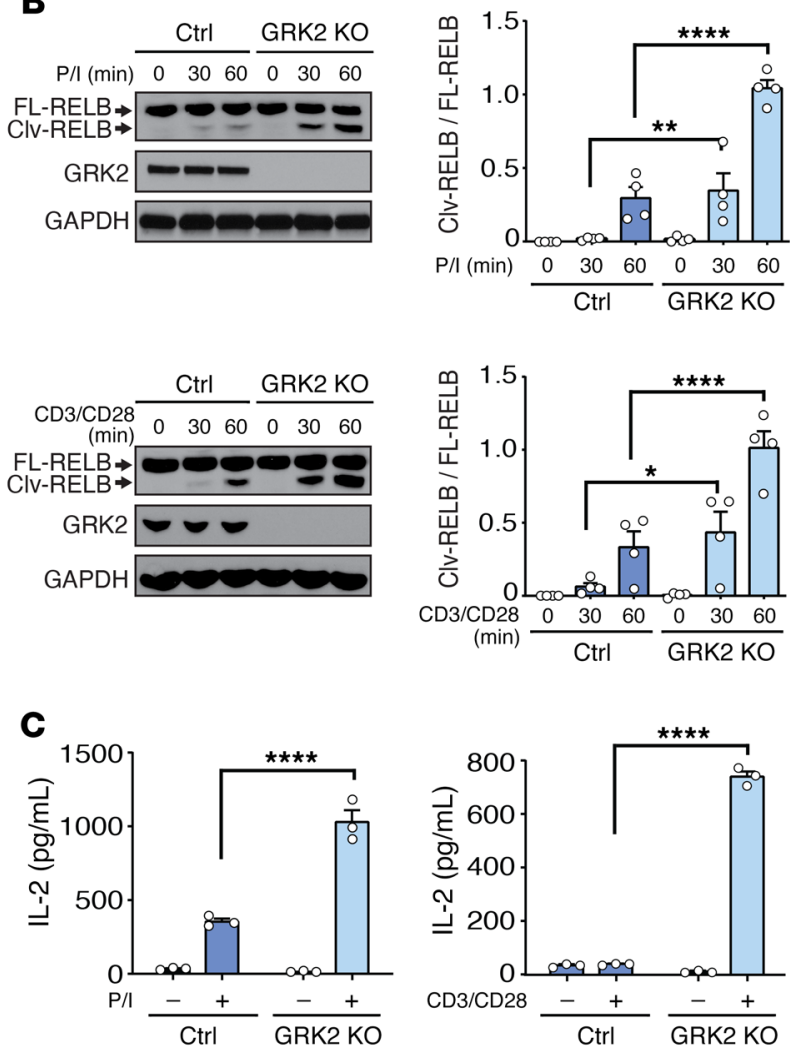
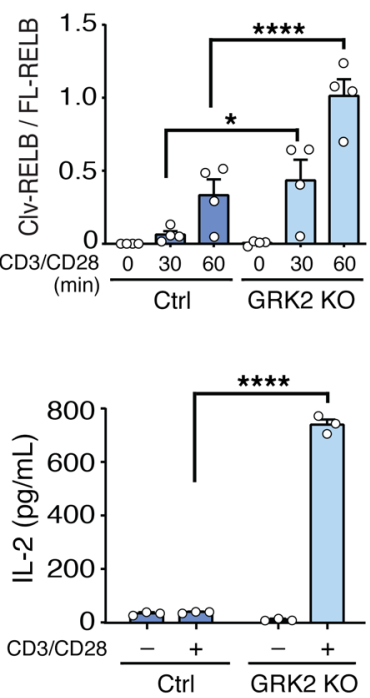

D

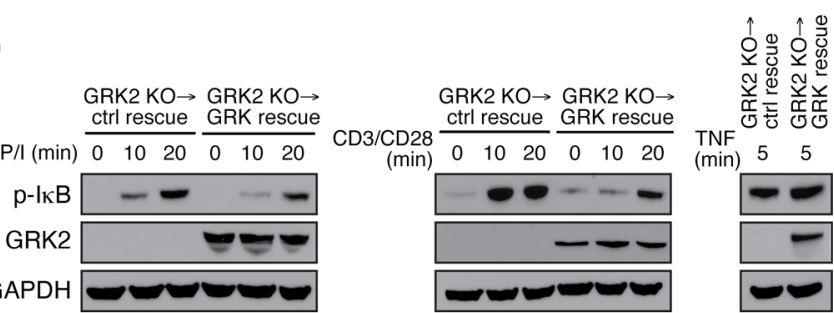

E

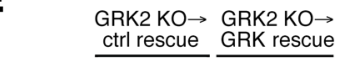

$P / I(\min ) \frac{\text { ctrl rescue }}{0 \quad 30 \quad 60} \frac{\text { GRK rescue }}{0 \quad 30 \quad 60}$

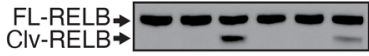

GRK2

-

GAPDH
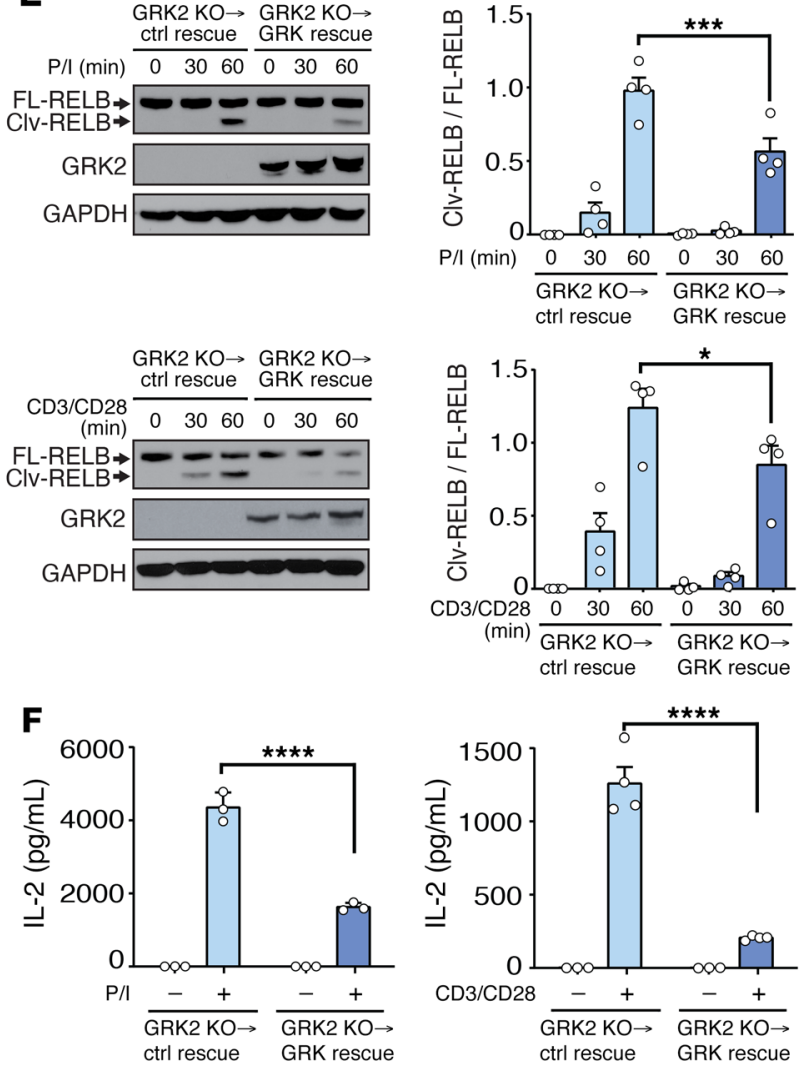

Figure 5. GRK2 CRISPR/Cas9 knockout leads to enhanced MALT1-dependent activities in Jurkat T cells, and rescue of GRK2 reverses this phenotype. (A) GRK2 knockout (KO) leads to increased IKB phosphorylation after P/I (left) or anti-CD3/CD28 (middle) but not after TNF (right) stimulation in Jurkat T cells. GRK2-KO Jurkat T cells were made using Cas9/gRNA. GRK2 knockout was confirmed by Western blot. Blots are representative of at least 3 experiments. (B) GRK2 KO in Jurkat T cells leads to increased cleavage of RELB after P/I (top) or anti-CD3/CD28 (bottom) stimulation. Blots shown are representative of $n=4$. Clv, cleaved; FL, full-length. Quantification of cleavage is shown to the right of the blots. ${ }^{*} P<0.05,{ }^{* *} P<0.01,{ }^{* * * *} P<0.0001$. (C) GRK2 KO leads to increased IL-2 production in Jurkat T cells after P/I (left) or anti-CD3/CD28 (right) stimulation. IL-2 secretion was determined by ELISA ( $n=3$ ). ${ }^{* * * *} P<$ 0.0001. (D) GRK2 rescue in GRK2-KO Jurkat T cells reverses the enhanced IKB phosphorylation after P/I (left) or anti-CD3/CD28 (middle) but has no effect on the TNF response (right). GRK2-KO Jurkat T cells were rescued using lentivirus expressing WT GRK2, and stable cell lines were made by selection using puromycin. GRK2 rescue was confirmed by Western blot. Blots are representative of at least 3 experiments. (E) Rescue of GRK2 in GRK2-KO Jurkat T cells reverses the enhanced cleavage of RELB caused by GRK2 KO. Blots shown are representative of $n=4$. Quantification of cleavage is shown to the right of the blots. ${ }^{*} P<0.05,{ }^{* *} P<0.001$. (F) GRK2 rescue leads to reduced IL-2 production in GRK2-KO Jurkat T cells. IL-2 secretion was determined by ELISA $(n=3-4)$. All values are represented as mean \pm SEM. ${ }^{* * *} P<0.0001$. Statistical significance for $\mathbf{B}, \mathbf{C}, \mathbf{E}$, and $\mathbf{F}$ was evaluated by 2 -way ANOVA, followed by Tukey's multiple-comparisons test.

in modulating the growth and survival of MALT1-dependent lymphomas. As a first step in evaluating such a role for GRK2 in these tumors, we used a published data set to compare mRNA levels of GRK2 (also known as ADRBK1) in 44 DLBCL patient tumor samples versus 20 healthy B cell control samples (51). We found that GRK2 mRNA levels are markedly lower in a subset of DLBCL cases compared with normal B cell controls (Figure 6A). We considered the possibility that the observed decrease in GRK2 could reflect gene expression changes during $B$ cell differentiation rather than a lymphoma/cancer-associated effect, but our analyses of publicly available data sets suggest that GRK2 mRNA levels remain relatively constant and high throughout B cell development (Figure 6B). Kaplan-Meier analysis of the publicly available Visco data set (GEO GSE31312) (52) showed that progression-free survival and overall 


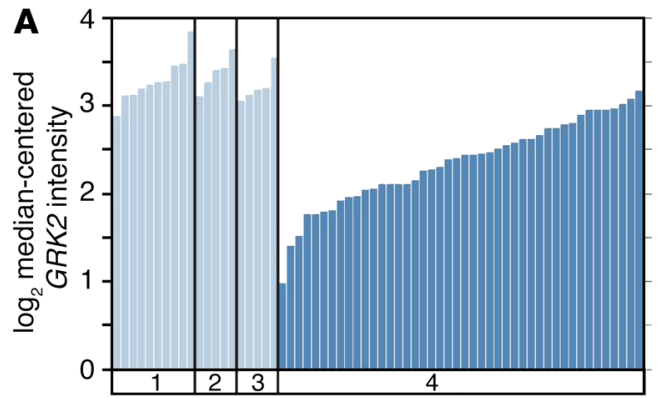

Cell-type category

\begin{tabular}{|c|l|}
\hline Category & Description \\
\hline $\mathbf{1}$ & Germinal center B lymphocyte \\
\hline $\mathbf{2}$ & Memory B lymphocyte \\
\hline $\mathbf{3}$ & Naive pregerminal B lymphocyte \\
\hline $\mathbf{4}$ & Diffuse large B cell lymphoma \\
\hline
\end{tabular}

B

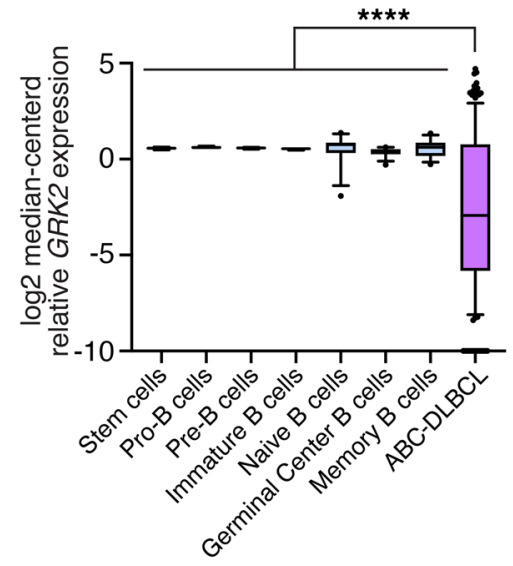

Figure 6. Lower GRK2 expression level is associated with reduced survival in ABC-DLBCL. (A) GRK2 mRNA levels are markedly lower in a subset of DLBCL tumor specimens ( $n=44)$ compared with normal control B cells $(n=20)$. Data were accessed using www. oncomine.com (GSE12195). GRK2 expression values were median centered and expressed on $\log _{2}$ scale. (B) GRK2 mRNA expression levels are relatively consistent throughout $B$ cell development and significantly higher in normal $B$ cells than what is seen in ABC-DL$B C L$ cells. Data were obtained from public repositories (GSE2350, GSE10846, GSE22886, and CSE45460) and analyzed using R (version 3.0.2) and GraphPad Prism (version 7.0.1). $P$ value was calculated by 1-way ANOVA, followed by Bonferroni post-test. ${ }^{* * *} P$ $<0.0001$. (C and $\mathbf{D}$ ) Rates of progression-free survival (PFS) (C) and overall survival (OS) (D) of $A B C-D L B C L$ patients are lower in patients with low GRK2 (bottom 25\%) compared with high GRK2 (top 25\%). Expression of GRK2 was stratified into high, mid, and low categories using data set-wide quartile cutoffs (low 25\%, mid 50\%, high 25\%). Statistical significance was evaluated using log-rank test. Data for $\mathbf{C}$ and $\mathbf{D}$ were accessed from a public repository (CSE31312).

survival rates for ABC-DLBCL are significantly worse in patients with low GRK2 levels (bottom 25\%) as compared with high GRK2 (top 25\%) (Figure 6, C and D). We also performed multivariate Kaplan-Meier analysis for progression-free survival and overall survival, controlling for age, sex, stage, prognostic index, and chemotherapy response, and results indicated that lower GRK2 level remains an independent predictor in ABC-DLBCL patients (representative graphs with correction for age and sex are shown in Supplemental Figure 5, A and B). The association of lower GRK2 expression level with inferior survival in ABC-DLBCL was also further confirmed by analysis of a second independent data set (GSE4732) (ref. 53 and Supplemental Figure 5, C and D). In contrast to ABC-DLBCL, GRK2-low groups do not show reduced survival relative to GRK2-high groups for patients with GCB-DLBCL, a DLBCL subtype that is not dependent on MALT1 for growth and survival (ref. 7 and Supplemental Figure 5, E and F).

We also compared GRK2 mRNA expression levels in ABC-DLBCL tumor specimens obtained from patients with localized disease with those from patients with advanced-stage disease. We found that lower GRK2 expression level is associated with an increased degree of extranodal spread of lymphoma (GSE10846) (Supplemental Figure 5G and ref. 54). Together, our analyses indicate that lower GRK2 level is associated with inferior survival in ABC-DLBCL.

GRK2 restrains MALT1-dependent signaling and cellular proliferation in $A B C-D L B C L$. Multiple studies have demonstrated that ABC-DLBCL cells are dependent on MALT1 proteolytic activity for growth and survival $(27,29)$. We queried publicly available cell line mRNA expression data sets and noted that most ABC-DLBCL cell lines demonstrated lower GRK2 mRNA expression as compared with non-MALT1-dependent GCB-DLBCL cell lines (Figure 7A and Supplemental Figure 6A). We next assessed GRK2 protein levels in several ABC-DLBCL cell lines, as well as in several GCB-DLBCL cell lines. Specifically, we evaluated HBL1, TMD8, and OCI-Ly10 cells, which harbor activating mutations in a BCR CD79 subunit, as well as OCI-Ly3 cells, which harbor an activating mutation in CARMA1 (ref. 21 and Figure 7B). Although a small cohort, we were intrigued to find that GRK2 protein level is quite low in a subset of ABC-DLBCL lines when compared with GCB-DLBCL lines, which is consistent with what we had observed above in cell line mRNA expression data sets. We evaluated the interaction of GRK2 and MALT1 in ABC-DLBCL cells and found that despite the relatively low GRK2 protein level and despite the presence of upstream activating mutations that drive constitutive CBM assembly and MALT1 activity, some degree of baseline interaction between GRK2 and MALT1 can still be detected by co-IP in both OCI-Ly3 and HBL1 cells (Supplemental Figure 6B). Intriguingly, the relative amount of GRK2 coimmunoprecipitated with MALT1 is notably higher in GCB cells (OCI-Ly1 and OCI-Ly7 cells: density ratios of GRK2/ MALT1 with MALT1 co-IP are 1.36 and 1.90, respectively) than in ABC-DLBCL cells (OCI-Ly3 and HBL1: GRK2/MALT1 density ratios are 0.41 and 0.23, respectively) (Supplemental Figure 6C). We speculate that this relative difference in GRK2-MALT1 interaction could be the result of some degree of GRK2 dissociation from MALT1 induced by the upstream gain-of-function mutations that mimic AgR stimulation in the ABC-DLBCL cells.

We attempted to overexpress GRK2 in HBL1 cells, our ABCDLBCL line with the lowest GRK2 mRNA and protein levels, using 
A

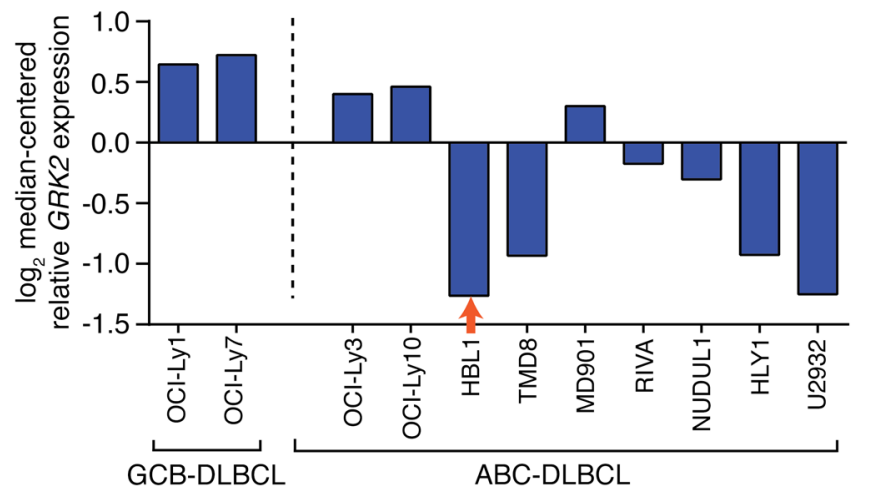

B

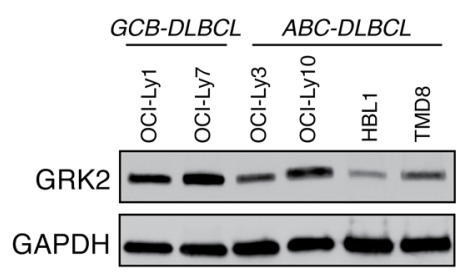

C

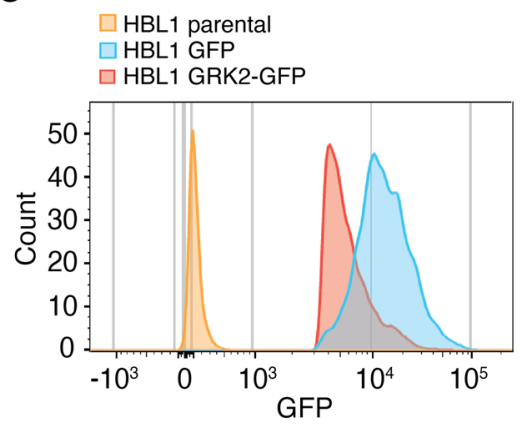

D

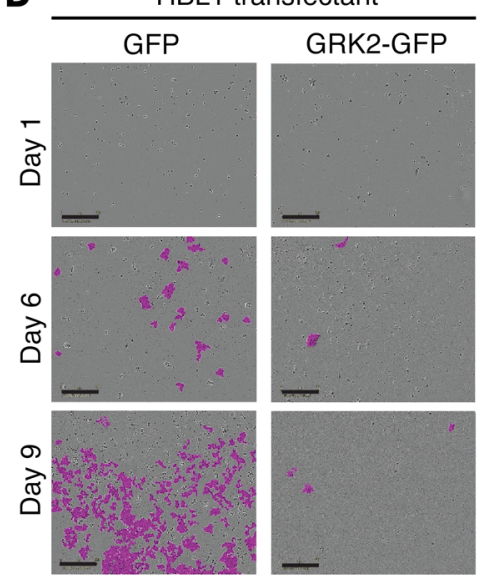

$\mathbf{E}$

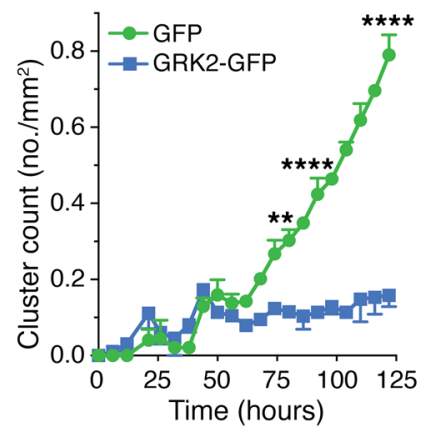

Figure 7. Overexpression of GRK2 inhibits ABC-DLBCL proliferation. (A) HBL1 cells demonstrate relatively low GRK2 mRNA expression. CDNA microarray data were retrieved from a public repository (ArrayExpress, E-GEOD-42203) and analyzed. DLBCL cell lines were grouped based on GCB or ABC subtype. (B) HBL-1 cells express the lowest level of GRK2 protein among all cell lines tested. GRK2 protein levels in a panel of GCB-DLBCL (OCI-Ly1, OCI-Ly7) and ABC-DLBCL (OCI-Ly3, OCI-Ly10, HBL1, and TMD8) cells were assessed by Western blot. Cell lysates were subjected to immunoblotting with antibodies as indicated. Blots are representative of 2 independent experiments. (C) GRK2-GFP is effectively expressed in HBL1 cells infected with lentivirus, as detected by flow cytometry. Data are representative of at least 5 independent experiments. (D) GRK2-GFP-positive cells fail to proliferate, while control GFP-only HBL1 cells proliferate efficiently. Proliferation of HBL1 cells was monitored using a cell clustering immune cell proliferation assay. Representative images of 3 independent experiments are shown. Phase-contrast images are overlaid with Incucyte Zoom (Essen Biosciences) confluence segmentation mask (magenta). Scale bars: $300 \mu \mathrm{m}$. (E) Cluster count quantification is shown with time as a continuous variable. HBL1 cell proliferation was measured at 5-hour intervals using Incucyte Zoom $(n=3)$. Two-way ANOVA and Šidák's multiple-comparisons test were performed to show growth difference between the GRK2-GFP and control GFP groups. ${ }^{* *} P<0.01,{ }^{* * *} P<0.0001$.

a lentiviral approach. Although we were able to isolate GFP-tagged GRK2-positive HBL1 cells by flow cytometry sorting (Figure 7C), these GRK2-overexpressing cells failed to proliferate, while control GFP-only-positive HBL1 cells proliferated efficiently (Figure 7, D and E). Similar to the situation with HBL1 cells, we were able to isolate GFP-tagged GRK2 positive OCI-Ly3 cells (Supplemental Figure 6D), and like the HBL1 cells expressing GFP-GRK2, these OCI-Ly3 cells expressing GFP-GRK2 did not survive. In contrast to these ABC-DLBCL cell lines, we were able to successfully generate GCB-DLBCL lines (OCI-Ly1 and OCI-Ly7) as well as Jurkat T cell lines that overexpress GFP-tagged GRK2 (Supplemental Figure $6 \mathrm{E})$ using the same lentiviral system. These results are consistent with the notion that GRK2 can function as a tumor modulator/suppressor, and its downregulation in a subset of ABC-DLBCL cases is required for pathologic growth and survival. Restoration of GRK2 expression in this setting therefore inhibits cell growth and survival.

In light of these results, we took the converse approach, and analyzed the impact of GRK2 knockdown in OCI-Ly3 cells, an ABC-DLBCL line with higher GRK2 level compared with HBL1 and others (Figure 7, A and B, and Supplemental Figure 6A). Using lentiviral shRNA, we generated multiple OCI-Ly3 clones with significantly reduced GRK2 levels, and these GRK2deficient OCI-Ly3 clones all displayed significantly enhanced basal IкB phosphorylation as compared with control OCI-Ly3 clones (Figure 8A, left). We also performed targeted deletion of GRK2 in OCI-Ly3 cells using CRISPR/Cas9. After selecting and expanding individual clones (designated as T5, T39, and T47), we confirmed the targeted disruption of GRK2 using Sanger sequencing (TIDE analysis and chromatogram sequence alignment are shown in Supplemental Figure 7). Similar to our analysis of GRK2-shRNA-knockdown OCI-Ly3 clones, the GRK2-CRISPR-knockout OCI-Ly3 clones demonstrated higher levels of basal IкB phosphorylation (Figure 8A, middle, and Supplemental Figure 8A). We next attempted to rescue the homozygous GRK2-CRISPR-knockout clone T5 using lentivirus expressing WT GRK2. While we were successful in expressing GRK2 in 
GRK2 shRNA KD (OCI-Ly3 clones)

A

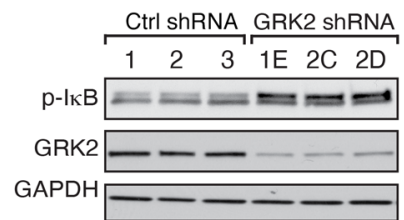

B

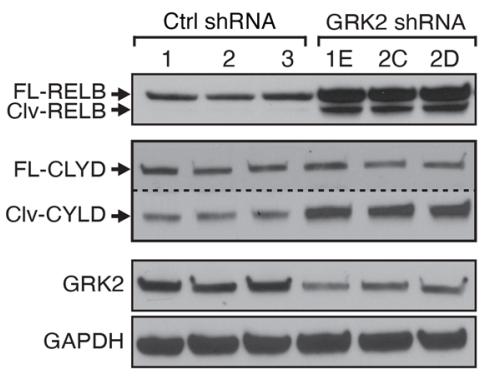

C

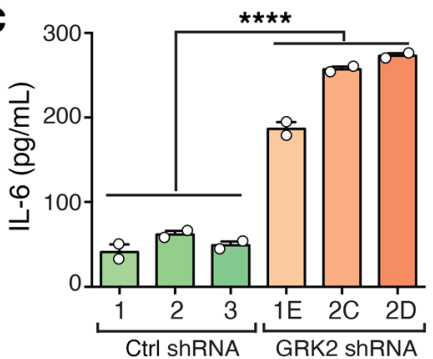

D

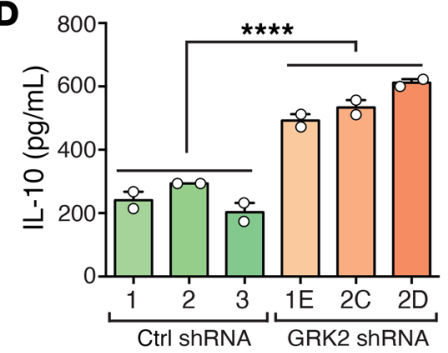

E

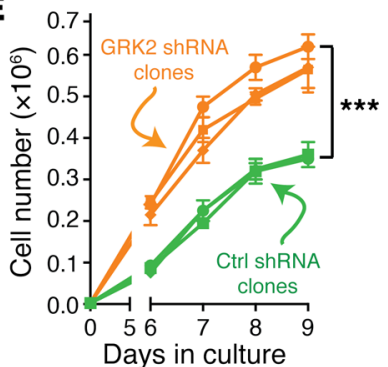

GRK2 CRISPR KO (OCl-Ly3 clone T5)
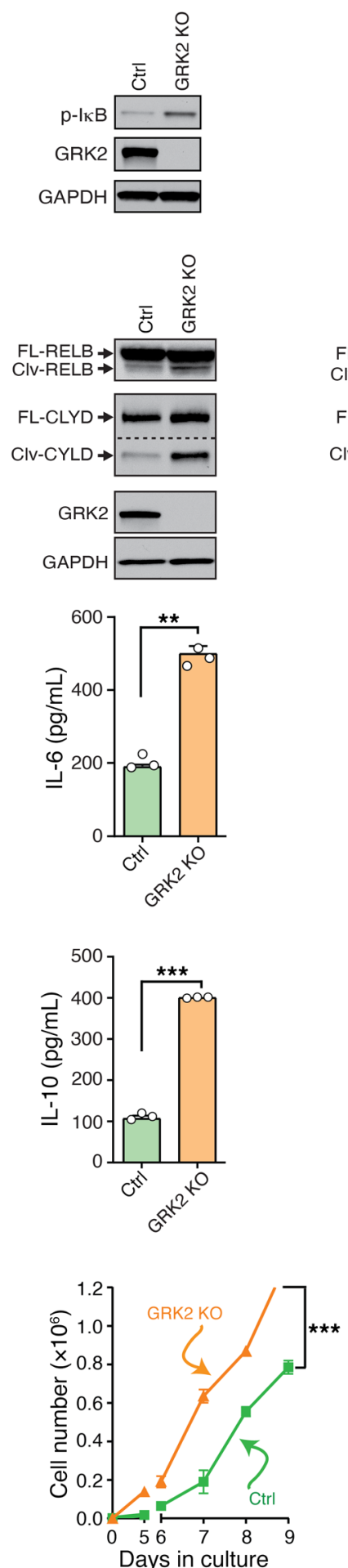

GRK2 rescue

(OCl-Ly3 clone T5)

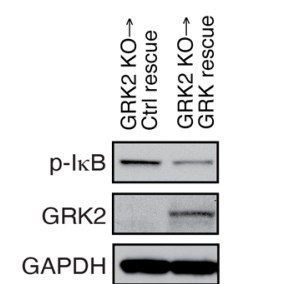

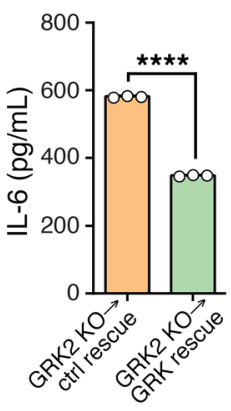
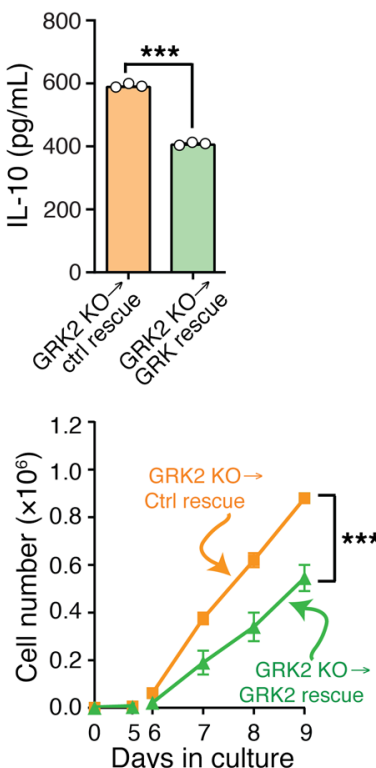

Figure 8. shRNA knockdown or CRISPR/Cas9 knockout of GRK2 in ABC-DLBCL (OCI-Ly3) cells leads to enhanced MALT1-mediated IKB phosphorylation, RELB and CYLD cleavage, cytokine secretion, and cell proliferation, and GRK2 rescue reverses these effects. (A) GRK2 knockdown (KD) (left) or knockout (KO) (middle) leads to increased basal I $\kappa B$ phosphorylation in ABC-DLBCL line OCI-Ly3. Stable OCI-Ly3 cells with GRK2 KD were made using specific shRNA lentivirus. GRK2 KOs were made using Cas9/ gRNA. GRK2 KD or KO was confirmed by Western blot. Rescue of GRK2 in GRK2-KO cells reverses this phenotype (right). Blots are representative of at least 3 experiments. (B) GRK2 KD (left) or KO (middle) in OCI-Ly3 cells leads to increased cleavage of RELB and CYLD. Rescue of GRK2 in GRK2-KO cells reverses this phenotype (right). Blots shown are representative of 3 experiments. Clv, cleaved; FL, fulllength. (C and D) GRK2 KD (left) leads to increased IL-6 and IL-10 production in OCI-Ly3 cells. Control clones (1, 2, and 3 ) and CRK2-KD clones (1E, 2C, and 2D) were analyzed separately (total of $n=$ 6 for both control and GRK2 KD). GRK2 $\mathrm{KO}$ (middle) leads to increased IL- 6 and IL-10 production in OCI-Ly3 cells ( $n=$ 3). Rescue of GRK2 in GRK2-KO cells (right) reverses this phenotype $(n=3)$. IL-6 and IL-10 secretion was determined by ELISA. All values are represented as mean \pm SEM. ${ }^{* *} P<0.01,{ }^{* *} P<$ $0.001,{ }^{* * * *} P<0.0001$, by unpaired, 2-tailed Student's $t$ test. (E) GRK2 KD (left) or KO (middle) leads to increased proliferation of OCl-Ly3 cells. Rescue of GRK2 in GRK2-KO cells reverses this phenotype (right) $(n=3)$. Two-way ANOVA and Šidák's multiple-comparisons test were performed to show proliferation differences between the GRK2-KD or -KO and control groups (only significance for the end time points is shown). ${ }^{* *} P<0.001$. our GRK2-CRISPR-knockout cells, the level of GRK2 expression achieved in the rescued OCI-Ly3 cells was significantly lower than the endogenous GRK2 level in CRISPR control cells (Supplemental Figure 8B). Even with this relatively lower level of GRK2 expression, we found that GRK2 rescue reversed the enhanced I $\mathrm{B}$ phosphorylation (Figure $8 \mathrm{~A}$, right).
Consistent with our previous analysis indicating that GRK2 inhibits MALT1 proteolytic activity (Figure 3), we found that the GRK2-shRNA-knockdown and GRK2-CRISPR-knockout OCILy3 clones demonstrated enhanced MALT1-mediated cleavage of RELB and CYLD compared with controls (Figure 8B and Supplemental Figure 8, C and D). Notably, GRK2 knockdown/knockout 


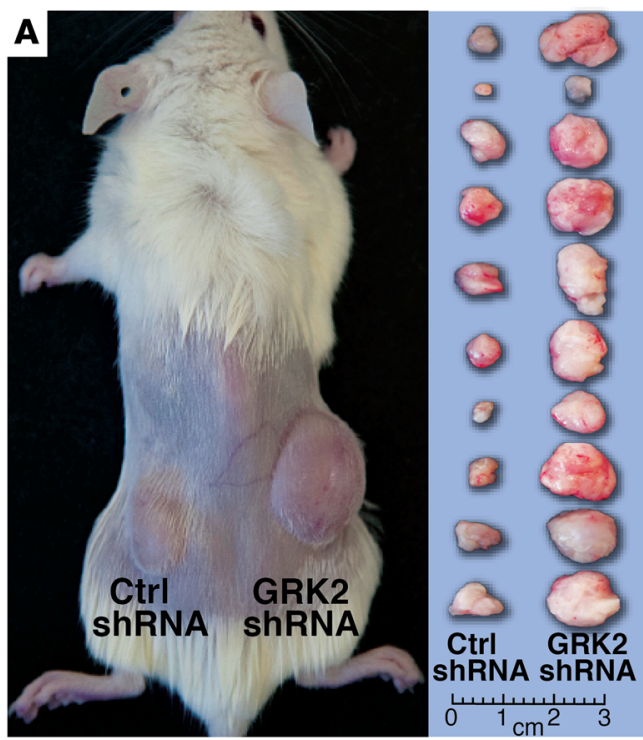

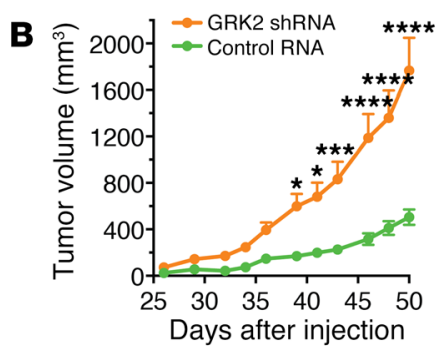

C

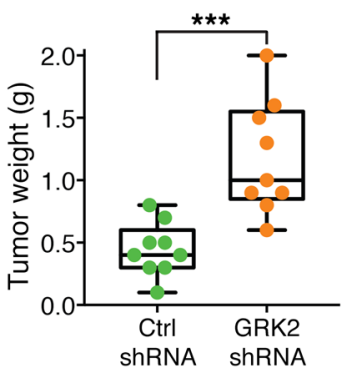

Figure 9. Knockdown of GRK2 leads to enhanced tumor growth of $A B C-D L B C L$ cells in vivo. (A-C) GRK2 knockdown leads to increased tumor volume and weight in a xenograft model of DLBCL. NOD. Cg-Prkdc ${ }^{\text {scid }} \| 2$ rg $^{\text {tmiWil }} / \mathrm{Sz}$ (NSG) mice were inoculated with OCI-Ly3 cells $\left(2.5 \times 10^{6}\right)$ stably expressing control shRNA or GRK2 shRNA. Tumors were measured 3 times weekly with calipers, and tumor weight was determined at week 7 . A representative mouse and resected tumor pairs $(n=10)$ are shown in A. Twoway ANOVA and Šidák's multiple-comparisons test were performed to show growth differences between the GRK2 shRNA and control groups. Paired $t$ test was performed for tumor weights. ${ }^{*} P<0.05$, ${ }^{* * *} P<0.001,{ }^{* * *} P<0.0001$ in ABC-DLBCL was associated with an increase in total RELB levels in these cells. It is possible that this occurs as a result of enhanced MALT1-dependent activation of the transcription of the $R E L B$ gene, a known NF- $\kappa \mathrm{B}$ transcriptional target (15). Importantly, GRK2 rescue reversed the enhanced RELB and CYLD cleavage in GRK2-knockout OCI-Ly3 cells (Figure 8B, right).

IL-6 and IL-10 are cytokines present in the lymphoma tumor microenvironment that promote the proliferation and survival of the malignant B cells (55). In DLBCL, autocrine IL-6 production provides proliferative and antiapoptotic signals, and elevated IL-6 levels in patient serum correlate with adverse outcome (56). Previous studies have demonstrated that ABC-DLBCL cells, including OCI-Ly3, spontaneously secrete IL-6 and IL-10 (55), and that transcription and expression of these 2 cytokines depend on MALT protease activity (26). Again, consistent with a tumor-suppressing role for GRK2, we found that GRK2 shRNA knockdown or CRISPR knockout enhanced the secretion of these tumor-promoting cytokines (Figure 8, C and D, and Supplemental Figure 8, E and F), and GRK2 rescue reversed this enhanced cytokine secretion (Figure 8, C and D).

The GRK2-deficient OCI-Ly3 clones all displayed significantly enhanced proliferation in vitro as compared with control OCILy3 clones (Figure 8E, left and middle, and Supplemental Figure $8 G$ ), and GRK2 rescue reversed this enhanced proliferation (Figure $8 \mathrm{E}$, right). Notably, stable knockdown of GRK2 in another ABC-DLBCL cell line, HBL1, had similar effects, enhancing basal I $\kappa$ B phosphorylation, MALT1 cleavage activity, IL-10 secretion, and in vitro proliferation (Supplemental Figure 9, A-E). Intriguingly, we observed that GRK2 knockdown in ABC-DLBCL cells also resulted in increased cell aggregation and attachment (Supplemental Figure 8H). It has been reported that MALT1-dependent cleavage of BCL10 enhances $\beta_{1}$ integrin-dependent T cell adhesion (17). Our results in ABC-DLBCL cells could therefore be consistent with a model in which a decrease in GRK2 level results in an increase in MALT1-dependent cleavage of BCL10, leading to enhanced cell adhesion. Further studies are needed to more fully elucidate the molecular mechanisms mediating this observed increase in adhesion in the GRK2-deficient cells.
In light of our in vitro observations, we next examined the influence of GRK2 on lymphoma growth in vivo. To this end, we used our OCI-Ly3 ABC-DLBCL cell lines harboring either control shRNA or GRK2-targeting shRNA, and subcutaneously injected these lines into opposite flanks of 5-week-old NOD.Cg-Prkdc ${ }^{\text {scid }}$ Il2rg ${ }^{\text {tm1Wjl }} /$ SzJ (NSG) mice. Xenograft growth was monitored over the ensuing 2 months, revealing a dramatic increase in the growth of tumors with GRK2 knockdown (Figure 9, A-C). Thus, both our in vitro and in vivo studies provided evidence that GRK2 abrogates ABC-DLBCL tumor growth.

\section{Discussion}

Our present study reveals a new mechanism by which MALT1, a proto-oncoprotein that plays a critical role in the pathogenesis of several subtypes of NHL, is regulated. The MALT1 gene was originally identified in 1999 as a target for chromosomal translocation in the B cell malignancy MALT lymphoma (57-60). Since that time, accumulating evidence has revealed that the CBM complex plays an essential role in the normal adaptive immune response by bridging TCR or BCR upstream signaling to the canonical NF- $\mathrm{BB}$ pathway, thus promoting lymphocyte activation and proliferation in response to antigen $(47,61,62)$. Deregulation of this CBM complex, with resultant constitutive MALT1 activity and unrestricted $\mathrm{NF}-\kappa \mathrm{B}$ signaling, has recently emerged as a common feature of multiple lymphoid malignancies (7). Here, we demonstrate that GRK2 binds to and inhibits MALT1, negatively impacting both MALT1 scaffolding and proteolytic activities and blocking downstream MALT1-mediated NF- $\kappa$ B activation.

Several groups have demonstrated that ABC-DLBCL, which can harbor gain-of-function mutations in CARMA1 or the upstream CD79A or CD79B subunit of BCR, relies on constitutive MALT1 activity for growth and survival $(21,23,26,28)$. We provide multiple lines of evidence indicating that GRK2 inhibits the growth of these MALT1-dependent tumors. More recently, Rahal et al. demonstrated that a subset of mantle cell lymphomas similarly depend on constitutive MALT1 signaling for growth and survival (34). In addition, a role for deregulated MALT1 signaling has 
recently been extended beyond $\mathrm{B}$ cell lymphoma to include $\mathrm{T}$ cell malignancy. Activating mutations in CARMA1, and its upstream regulators PKC $\beta$ and PLC $\gamma 1$, have been identified in both adult T cell leukemia/lymphoma and Sézary syndrome, a form of cutaneous $\mathrm{T}$ cell lymphoma, suggesting that these malignant $\mathrm{T}$ cells are also dependent on MALT1 for survival (63-65). This ever-expanding role for MALT1 in a spectrum of lymphoid malignancy suggests that inhibition of MALT1 by GRK2 likely impacts the pathogenesis of multiple subtypes of lymphoma beyond ABC-DLBCL. This concept underscores the potentially broad scope of influence of the GRK2-MALT1 interaction in lymphoid malignancy and warrants further study.

The discovery that in addition to scaffolding activity, MALT1 also possesses proteolytic activity, represented a major step in advancing our understanding of lymphocyte biology and the molecular pathogenesis of CBM-driven lymphomas (17, 18, 66). Thus far, 10 specific proteolytic substrates of MALT1 have been identified $(20,67)$, and we demonstrate that GRK2 inhibits MALT1-mediated cleavage of 2 of these known substrates, RELB and CYLD. MALT1 protease activity is thought to sustain $\mathrm{NF}-\kappa \mathrm{B}$ activation and promote lymphomagenesis in part by cleaving RELB, an NF- $\kappa \mathrm{B}$ subunit that forms transcriptionally inactive complexes with and thereby inhibits RELA and c-REL (15). While modulation of NF- $\kappa$ B activity is the most well-studied role of MALT1 protease activity, MALT1 cleaves several additional substrates that impact other aspects of lymphocyte biology. For example, MALT1-dependent cleavage of the deubiqitinase CYLD is essential for AgR-induced activation of JNK (19), and similarly to constitutive NF- $\kappa \mathrm{B}$ activation, constitutive JNK activation is also a hallmark of ABC-DLBCL (68). In addition, recent studies demonstrate that MALT1 cleaves multiple substrates involved in regulating RNA stability in lymphocytes $(69,70)$. MALT1 is also able to cleave its binding partner BCL10, and this cleavage may promote lymphocyte adhesion (17). We expect that, in addition to inhibiting cleavage of CYLD and RELB, as shown here, GRK2 likely influences the processing of other diverse MALT1 proteolytic substrates. Precisely how GRK2 influences the overall balance of MALT1-driven NF- $\kappa$ B activity, JNK signaling, RNA stability, cellular adhesion, and/or other cellular functions impacted by MALT1 protease activity probably varies depending on cellular context, and the broad impact of GRK2 on these processes warrants further investigation. Our current study indicates that in MALT1dependent ABC-DLBCL cells, a net effect of GRK2 is to inhibit cell aggregation and proliferation.

Our data show that AgR stimulation induces the dissociation of GRK2 and MALT1, and this occurs within the same general time course as AgR-induced CARMA1 association with BCL10/MALT1 and phosphorylation of I $\kappa$ B. We also find that GRK2 knockdown results in significantly enhanced AgR-induced CARMA1 association with BCL10/MALT1 and I $\mathrm{B}$ phosphorylation. Together these results suggest that GRK2 inhibits overall CBM complex assembly. While we find that overexpression of either GRK2 or the GRK2 N-terminal fragment $(\alpha \mathrm{N} / \mathrm{RH}$, aa 1-173) inhibits the coimmunoprecipitation of MALT1 and BCL10 as well as downstream BCL10/MALT1-dependent NF- $\kappa$ B activation, we do not observe an increase in BCL10-MALT1 interaction when GRK2 is knocked down in T cells. We do find that MALT1 scaffolding activity (mea-

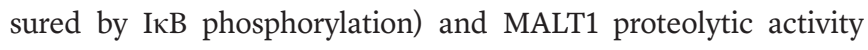
(measured by substrate cleavage), as well as MALT1-dependent upregulation of IL-2, are all significantly increased in cells with GRK2 knockdown. Thus, based on our analysis thus far, it seems likely that the ability of GRK2 to inhibit BCL10/MALT1-dependent $\mathrm{NF}-\kappa \mathrm{B}$ signaling is not solely a result of direct competition between BCL10 and GRK2 for MALT1 binding. In fact, we find that GRK2 inhibits the proteolytic activity of purified MALT1 in vitro, which indicates that GRK2 can exert an inhibitory effect on MALT1 activity in the absence of either CARMA1 or BCL10. Detailed structural studies will be needed in the future in order to elucidate the precise molecular mechanism(s) by which GRK2 interaction with MALT1 results in the inhibition of MALT1 signaling activities. Importantly, while this current article was in preparation, a detailed cryo-electron microscopy structural analysis of the BCL10-MALT1 interaction was published by the Krappmann and Lammens laboratories (44). This new work, which revealed the interaction of BCL10 with the MALT1 DD, will be highly relevant to future structural analyses of the interaction of GRK2 with the MALT1 DD.

Inhibition of MALT1 proteolytic activity is selectively toxic to ABC-DLBCL human lymphoma cell lines in vitro and to ABC-DLBCL xenograft tumors in vivo $(26,27,29)$. These findings have led to considerable interest in the development of inhibitors of MALT1 protease activity as therapeutic agents. Multiple distinct categories of small-molecule MALT1 protease inhibitors have been reported, including (a) phenothiazine derivatives (e.g., mepazine), which bind to an allosteric site located between the MALT1 Ig3 domain and catalytic domain, and reversibly inhibit MALT1 by preventing the rearrangement of the enzyme into an active conformation (29); (b) MI-2 and its derivatives, which are irreversible inhibitors thought to covalently modify the MALT1 active site cysteine (27, 71); (c) $\beta$-lapachone analogs, which are also irreversible inhibitors that form a covalent bond with the MALT1 catalytic cysteine (72); and (d) MLT-748 and MLT-747, which are closely related allosteric inhibitors that bind to the interface between the catalytic and Ig3 domains (73). Our current study suggests that GRK2 inhibits MALT1 proteolytic activity via a mechanism distinct from that of these MALT1 protease inhibitors, by interacting with the MALT1 DD. We have determined that the N-terminal region of GRK2, composed of a short helix $(\alpha \mathrm{N})($ aa 1-20) followed by a regulator of $\mathrm{G}$ protein signaling homology $(\mathrm{RH})$ protein-protein interaction domain (aa 30-173), mediates this interaction with MALT1. This region of GRK2 (aa 1-173), which excludes the kinase and PH domains, is both necessary and sufficient to inhibit MALT1-dependent signaling, indicating that GRK2 kinase activity is not required to inhibit MALT1. It will be of great interest to precisely define the structural features within GRK2 that are required for interaction with the MALT1 DD and inhibition of MALT1 activities. We anticipate that elucidation of these required features of GRK2 could provide a roadmap for developing a new category of MALT1 inhibitor that would abrogate both scaffolding and proteolytic activities via interaction with the MALT1 DD.

GRK2 is emerging as an important “oncomodulator," a protein that influences multiple cellular functions related to the hallmarks of cancer, such as cell proliferation, cell survival, cell motility, cell metabolism, and angiogenesis, via its impact on cancer-relevant signaling networks (38). Many recent studies show 
that in addition to its canonical role of regulating $G$ protein-coupled receptors via phosphorylation-dependent desensitization and internalization, GRK2 can also exert effects in a phosphorylation-independent manner by engaging in a diverse repertoire of protein-protein interactions $(36,74)$. GRK2 has been reported to influence tumor progression in several different cancers, and the specific mechanism by which GRK2 exerts its influence depends on the tumor cell type. For example, it has been reported that GRK2 promotes the growth of certain breast cancers via phosphorylation and activation of histone deacetylase 6 (HDAC6) $(38,75)$. As another example, 2 recent reports suggest that GRK2 is overexpressed in pancreatic cancer and may serve as an indicator of unfavorable prognosis $(76,77)$. In contrast to breast and pancreatic cancer, GRK2 appears to inhibit tumor growth in other cancers. For example, several studies suggest that GRK2 inhibits the proliferation of hepatocellular carcinoma (HCC) cells, and this is thought to occur via GRK2-mediated inhibition of insulin-like growth factor 1 (IGF-1) signaling $(78,79)$. In HCC, GRK2 kinase activity is required for GRK2 to suppress cell proliferation. Unlike this kinase-dependent mechanism by which GRK2 inhibits IGF-1 signaling and cell proliferation in HCC, our studies suggest that GRK2 inhibits MALT1-dependent tumor progression via a mechanism that is kinase-independent.

Our studies suggest that GRK2 functions as an oncomodulator/tumor suppressor in ABC-DLBCL. We demonstrate that lower GRK2 mRNA expression in ABC-DLBCL, but not in GCB-DLBCL, is associated with worse overall survival and progression-free survival. Furthermore, our studies show that lowering GRK2 protein levels within ABC-DLBCL cells results in enhanced tumor growth in vitro and in vivo. These data lead us to propose that measuring GRK2 mRNA and/or protein levels in ABC-DLBCL tumor specimens might become useful in guiding prognosis and treatment. Additionally, it will be of great interest to elucidate the molecular mechanisms responsible for regulating levels of GRK2 mRNA and/ or protein and the degree of GRK2-mediated MALT1 inhibition in these tumors. Thus far, we have not identified specific mutations in the GRK2 gene that could account for the lower GRK2 mRNA expression in a subset of ABC-DLBCL tumor specimens or the lower GRK2 protein expression in a subset of ABC-DLBCL cell lines. However, GRK2 protein expression and function are known to be regulated at multiple levels, including at the posttranscriptional and posttranslational levels $(37,80,81)$. Future studies will be aimed at elucidating the mechanisms by which GRK2 expression and function are controlled in ABC-DLBCL and other MALT1-dependent tumors.

Taken together, our data identify GRK2 as a binding partner and negative modulator of MALT1. We found that GRK2 bound to the MALT1 DD, and, to our knowledge, GRK2 is the first protein demonstrated to inhibit MALT1 scaffolding and protease activities. Our molecular biological analyses of ABC-DLBCL cells and bioinformatic analyses of human patient tumor specimens are consistent with a tumor suppressor role for GRK2 in ABC-DLBCL, a cancer that requires active MALT1 for growth and survival. Since the MALT1 proto-oncoprotein is now emerging as a critical mediator of tumor growth and survival for an even wider variety of lymphoid neoplasms, elucidating the molecular mechanisms by which GRK2 inhibits MALT1 will be critical for understanding the overall land- scape of lymphoid neoplasia and for developing targeted therapies for the spectrum of MALT1-dependent malignancies.

\section{Methods}

Cell lines and reagents. The DLBCL cell lines OCI-Ly1, OCI-Ly3, OCI-Ly7, and OCI-Ly10 were provided by Mark D. Minden (University Health Network, Toronto, Ontario, Canada). HBL1 and TMD8 were provided by Louis Staudt (National Cancer Institute, NIH). OCI-Ly1 and OCILy7 cells were cultured in IMDM supplemented with 10\% FBS and 100 $\mathrm{U} / \mathrm{mL}$ penicillin/streptomycin. OCI-Ly3 cells were cultured in IMDM supplemented with $20 \% \mathrm{FBS}$ and $100 \mathrm{U} / \mathrm{mL}$ penicillin/streptomycin. OCI-Ly10 cells were cultured in IMDM with 20\% human serum (Valley Biomedical), penicillin/streptomycin, and $50 \mu \mathrm{M} \beta$-mercaptoethanol. HBL1, TMD8, Jurkat, and BJAB cells were cultured in RPMI 1640 medium with $10 \% \mathrm{FBS}$ and $100 \mathrm{U} / \mathrm{mL}$ penicillin/streptomycin. Stimulation of Jurkat and BJAB cells was accomplished by addition of phorbol 12-myristate 13 -acetate (PMA) $(5 \mathrm{ng} / \mathrm{mL}$; Sigma) and ionomycin $(1 \mu \mathrm{M}$; Calbiochem). GST-GRK2, GST-MALT1, and GST-BCL10 recombinant proteins were purchased from Novus Biologicals. TRC lentiviral human GRK2/ADRBK1 shRNA (TRCNO000000557, TRCNO000000558, TRCN0000000559, TRCN0000000560, TRCNO000000561) (NC0765786) were purchased from Dharmacon. Lentiviral ORF cDNA clones for GRK2/ADRBK1 (EX-A0311-Lv103 with N-eGFP) and empty vector control plasmid (EX-NEG-Lv103) were from GeneCopoeia. The proteasome inhibitor MG132 was purchased from Calbiochem. Pierce protein A/G-Agarose and Aminolink coupling resin were from Thermo Fisher Scientific. Plasmids expressing MALT1 and BCL10 have been described previously (43). pcDNAGRK2, pcDNAGRK2-K220R, pHA-GRK2, pHA-GRK2RH, and Flag-RelB plasmids were previously described $(15,82-84)$. FLAG-tagged CYLD expression plasmids were generated by cloning of CYLD cDNA, or fragments thereof, into the pEAK10 plasmid as previously described (85).

Recombinant protease cleavage assay. Recombinant GST-MALT1 was purified from E. coli. For measurement of protease activity in vitro, proteins were incubated for 3 hours at $30^{\circ} \mathrm{C}$ with $50 \mu \mathrm{M}$ Ac-LVSR-AMC (MCA-3952-PI; Peptides International), and the protease activity of MALT1 was determined by measurement of the increase of AMC fluorescence with a Synergy microplate reader (BioTek) as previously reported (86).

FRET-based assay of protease and flow cytometry. HEK293T cells were transfected with the appropriate constructs together with the eYFP-LeuVal-Ser-Arg-eCFP reporter construct. Twenty-four hours after transfection, cells were resuspended in flow cytometry buffer and analyzed with a BD LSR II flow cytometer (BD Biosciences) as previously reported (87).

Xenograft model of DLBCL. Tumors were engrafted into 5-weekold female NOD.Cg-Prkdc ${ }^{\text {scid }} \mathrm{Il} 2 \mathrm{rg}^{\text {tmiwil }} / \mathrm{SzJ}$ (NSG) mice from The Jackson Laboratory (stock 005557) by subcutaneous injection of $2.5 \times 10^{6}$ OCI-Ly3 control or GRK2 shRNA cells resuspended in Matrigel (Cultrex Basement Membrane Extract, Type 3, PathClear; Trevigen). Control and GRK2-knockdown tumors were engrafted on opposite flanks of individual mice, with 10 mice in each group. Tumor sizes were measured 3 times weekly after visual appearance using a caliper and calculated using the formula: (smallest diameter ${ }^{2} \times$ largest diameter) $/ 2$. Mice were sacrificed when tumors reached $2500 \mathrm{~mm}^{3}$.

Study approval. All experiments were performed in compliance with guidelines of the University of Pittsburgh and UCSF and according to the protocol approved by the IACUCs of these institutions. 
Statistical analysis. All values are represented as mean \pm SEM. Data were analyzed and statistics calculated with GraphPad Prism software using Student's $t$ test (for comparing 2 groups) and ANOVA (for comparing 3 or more groups). Figure legends contain details regarding $t$ test and ANOVA analyses for individual experiments. In vitro and in vivo growth curves were evaluated using 2-way ANOVA and Šidák's multiple-comparisons tests. Survival analysis was performed using the Kaplan-Meier method, and differences were compared using a log-rank (Mantel-Cox) test. Multivariate Kaplan-Meier analysis was performed using IBM Statistics SPSS 19 software and the Cox proportional hazards regression model. $P$ values of 0.05 or less were considered statistically significant.

Additional methods. Additional materials and methods are described in the Supplemental Methods.

\section{Author contributions}

JC designed and performed the majority of experiments, wrote the first draft of the manuscript, and constructed figures. LRK designed and performed analysis of Jurkat $\mathrm{T}$ cells with shRNA-GRK2 knockdown and CRISPR/Cas9-GRK2 knockout. NEH performed bioinformatic analyses of human tumor specimens. $\mathrm{MZ}$ and RS designed and performed experiments demonstrating GRK2 inhibition of MALT1 proteolytic activity. LMM designed and performed co-IP analyzing the impact of GRK2(1-173) on BCL10MALT1 interaction. HBK designed and performed ELISA analysis of cytokine secretion. HK designed and performed initial immunoprecipitation analyses to demonstrate GRK2-MALT1 interaction. VJC assisted with bioinformatic analysis and generation of GRK2-knockdown cell lines. PCD performed initial experiments demonstrating GRK2 inhibition of MALT1-mediated NF- $\mathrm{B}$ activation. EVD and MAM performed analysis of GRK2-deficient primary murine B cells under the supervision of JGC. MB generated multiple critical reagents required to investigate the interaction of GRK2 with the MALT1 DD. JGC developed a mouse strain with GRK2-deficient B cells and supervised analysis of primary murine B cells. NP performed the initial immunoprecipitation/mass spectrometry analysis that led to discovery of the GRK2-MALT1 interaction. MT developed methods for demonstrating GRK2-mediated inhibition of MALT1 protease activity, supervised those studies, and provided expert guidance for overall project development. PCL and LMML are the principal investigators of the laboratory where they supervised the development of this project in its entirety, procured funding to support the work, and led the construction of the manuscript. LMML is listed last because she serves as primary mentor for first author JC.

\section{Acknowledgments}

We thank the members of the Lucas/McAllister laboratory for their support. We thank Mark D. Minden and Louis Staudt for DLBCL cell lines. We thank Marzenna Blonska (University of Miami) for expert advice on establishing DLBCL xenografts. We thank the Flow Cytometry, Animal Care, and DNA Sequencing Core Facilities at the John G. Rangos Research Center at UPMC Children's Hospital of Pittsburgh (CHP), University of Pittsburgh School of Medicine, for expert technical assistance. We thank the Conover family for support of HK by a Conover Scholar Award. MT acknowledges support from the Swiss National Science Foundation, the Swiss Cancer League, and the Emma Muschamp Foundation. This work was supported by gifts from the Mario Lemieux Foundation/ Mario Lemieux Lymphoma Center for Children and Young Adults. We thank the CHP Foundation for outstanding fundraising efforts. This work was supported by grants to LMML from the University of Pittsburgh Physicians Foundation and the NIH (R01 CA124540). LMML is supported in part by a University of Pittsburgh Hillman Cancer Center Support Grant (5P30 CA047904). PCL is supported in part by funds from the UPMC Hillman Cancer Center.

Address correspondence to: Linda M. McAllister-Lucas or Peter C. Lucas, Rangos Research Building, Children's Hospital of Pittsburgh, University of Pittsburgh School of Medicine, 4401 Penn Avenue, Pittsburgh, Pennsylvania 15224, USA. Phone: 412.692.7608; Email: linda.mcallister@chp.edu; lucaspc@upmc.edu.
1. Roschewski M, Staudt LM, Wilson WH. Diffuse large B-cell lymphoma-treatment approaches in the molecular era. Nat Rev Clin Oncol. 2014;11(1):12-23.

2. Surveillance, Epidemiology, and End Results Program; National Cancer Institute Web Site. https://seer.cancer.gov. Updated May 30, 2019. Accessed December 23, 2019.

3. Alizadeh AA, et al. Distinct types of diffuse large B-cell lymphoma identified by gene expression profiling. Nature. 2000;403(6769):503-511.

4. Davis RE, Brown KD, Siebenlist U, Staudt LM. Constitutive nuclear factor kappaB activity is required for survival of activated $B$ cell-like diffuse large B cell lymphoma cells. J Exp Med. 2001;194(12):1861-1874.

5. Rosenwald A, et al. The use of molecular profiling to predict survival after chemotherapy for diffuse large-B-cell lymphoma. N Engl JMed. 2002;346(25):1937-1947.

6. Ngo VN, et al. A loss-of-function RNA interference screen for molecular targets in cancer. Nature. 2006;441(7089):106-110.
7. Juilland M, Thome M. Role of the CARMA1/ BCL10/MALT1 complex in lymphoid malignancies. Curr Opin Hematol. 2016;23(4):402-409.

8. Yang C, David L, Qiao Q, Damko E, Wu H. The CBM signalosome: potential therapeutic target for aggressive lymphoma? Cytokine Growth Factor Rev. 2014;25(2):175-183.

9. Noels $\mathrm{H}$, et al. A novel TRAF6 binding site in MALT1 defines distinct mechanisms of NF- $\kappa \mathrm{B}$ activation by API2-MALT1 fusions. J Biol Chem . 2007;282(14):10180-10189.

10. Oeckinghaus A, et al. Malt1 ubiquitination triggers NF-kappaB signaling upon T-cell activation. EMBO J. 2007;26(22):4634-4645.

11. Sun L, Deng L, Ea CK, Xia ZP, Chen ZJ. The TRAF6 ubiquitin ligase and TAK1 kinase mediate IKK activation by BCL10 and MALT1 in T lymphocytes. Mol Cell. 2004;14(3):289-301.

12. Lucas PC, McAllister-Lucas LM, Nunez G. NF-kappaB signaling in lymphocytes: a new cast of characters. J Cell Sci. 2004;117(pt 1):31-39.

13. Afonina IS, Elton L, Carpentier I, Beyaert R. MALT1-a universal soldier: multiple strategies to ensure NF- $\kappa \mathrm{B}$ activation and target gene expression. FEBS J. 2015;282(17):3286-3297.

14. McAllister-Lucas LM, Baens M, Lucas PC. MALT1 protease: a new therapeutic target in B lymphoma and beyond? Clin Cancer Res. 2011;17(21):6623-6631.

15. Hailfinger S, et al. Malt1-dependent RelB cleavage promotes canonical NF-kappaB activation in lymphocytes and lymphoma cell lines. Proc Natl Acad Sci U S A. 2011;108(35):14596-14601.

16. Klei LR, et al. MALT1 Protease activation triggers acute disruption of endothelial barrier integrity via CYLD cleavage. Cell Rep. 2016;17(1):221-232.

17. Rebeaud F, et al. The proteolytic activity of the paracaspase MALT1 is key in T cell activation. Nat Immunol. 2008;9(3):272-281.

18. Coornaert B, et al. T cell antigen receptor stimulation induces MALT1 paracaspase-mediated cleavage of the NF- $\kappa \mathrm{B}$ inhibitor A2O. Nat Immunol. 2008;9(3):263-271.

19. Staal J, et al. T-cell receptor-induced JNK activation requires proteolytic inactivation of CYLD by MALT1. EMBO J. 2011;30(9):1742-1752. 
20. Jaworski M, Thome M. The paracaspase MALT1: biological function and potential for therapeutic inhibition. Cell Mol Life Sci. 2016;73(3):459-473.

21. Davis RE, et al. Chronic active B-cell-receptor signalling in diffuse large B-cell lymphoma. Nature. 2010;463(7277):88-92.

22. Shaffer AL, Young RM, Staudt LM. Pathogenesis of human B cell lymphomas. Annu Rev Immunol. 2012;30:565-610.

23. Lenz G, et al. Oncogenic CARD11 mutations in human diffuse large B cell lymphoma. Science. 2008;319(5870):1676-1679.

24. Lohr JG, et al. Discovery and prioritization of somatic mutations in diffuse large B-cell lymphoma (DLBCL) by whole-exome sequencing. Proc Natl Acad Sci U S A. 2012;109(10):3879-3884.

25. Hailfinger S, Lenz G, Thome M. Targeting B-cell lymphomas with inhibitors of the MALT1 paracaspase. Curr Opin Chem Biol. 2014;23:47-55.

26. Ferch U, et al. Inhibition of MALT1 protease activity is selectively toxic for activated B celllike diffuse large B cell lymphoma cells. J Exp Med. 2009;206(11):2313-2320.

27. Fontan L, et al. MALT1 small molecule inhibitors specifically suppress ABC-DLBCL in vitro and in vivo. Cancer Cell. 2012;22(6):812-824.

28. Hailfinger S, et al. Essential role of MALT1 protease activity in activated B cell-like diffuse large B-cell lymphoma. Proc Natl Acad Sci U S A. 2009;106(47):19946-19951.

29. Nagel D, et al. Pharmacologic inhibition of MALT1 protease by phenothiazines as a therapeutic approach for the treatment of aggressive ABC-DLBCL. Cancer Cell. 2012;22(6):825-837.

30. Lucas PC, et al. A dual role for the API2 moiety in API2-MALT1-dependent NF- $\kappa \mathrm{B}$ activation: heterotypic oligomerization and TRAF2 recruitment. Oncogene. 2007;26(38):5643-5654.

31. Rosebeck S, et al. Cleavage of NIK by the API2-MALT1 fusion oncoprotein leads to noncanonical NF-kappaB activation. Science. 2011;331(6016):468-472.

32. Rosebeck S, et al. The API2-MALT1 fusion exploits TNFR pathway-associated RIP1 ubiquitination to promote oncogenic NF- $\kappa \mathrm{B}$ signaling. Oncogene. 2014;33(19):2520-2530.

33. Arora M, Kaul D, Varma N, Marwaha RK. Cellular proteolytic modification of tumor-suppressor CYLD is critical for the initiation of human T-cell acute lymphoblastic leukemia. Blood Cells $\mathrm{Mol}$ Dis. 2015;54(1):132-138.

34. Rahal R, et al. Pharmacological and genomic profiling identifies NF- $\mathrm{BB}$-targeted treatment strategies for mantle cell lymphoma. Nat Med. 2014;20(1):87-92.

35. Vallois $\mathrm{D}$, et al. Activating mutations in genes related to TCR signaling in angioimmunoblastic and other follicular helper T-cell-derived lymphomas. Blood. 2016;128(11):1490-1502.

36. Evron T, Daigle TL, Caron MG. GRK2: multiple roles beyond $G$ protein-coupled receptor desensitization. Trends Pharmacol Sci. 2012;33(3):154-164.

37. Penela P, Murga C, Ribas C, Lafarga V, Mayor F. The complex $G$ protein-coupled receptor kinase 2 (GRK2) interactome unveils new physiopathological targets. Br J Pharmacol. 2010;160(4):821-832.

38. Nogués L, Reglero C, Rivas V, Neves M, Penela P, Mayor F. G-protein-coupled receptor kinase 2 as a potential modulator of the hallmarks of cancer. Mol Pharmacol. 2017;91(3):220-228.

39. Vroon A, Heijnen CJ, Kavelaars A. GRKs and arrestins: regulators of migration and inflammation. J Leukoc Biol. 2006;80(6):1214-1221.

40. Matsumoto R, et al. Phosphorylation of CARMA1 plays a critical role in $\mathrm{T}$ cell receptor-mediated NF- $\kappa$ B activation. Immunity. 2005;23(6):575-585.

41. Sommer K, et al. Phosphorylation of the CARMA1 linker controls NF-kappaB activation. Immunity. 2005;23(6):561-574.

42. Langel FD, Jain NA, Rossman JS, Kingeter LM, Kashyap AK, Schaefer BC. Multiple protein domains mediate interaction between Bcl10 and MALT1. J Biol Chem. 2008;283(47):32419-32431.

43. Lucas PC, et al. Bcl10 and MALT1, independent targets of chromosomal translocation in malt lymphoma, cooperate in a novel NF- $\kappa \mathrm{B}$ signaling pathway. J Biol Chem. 2001;276(22):19012-19019.

44. Schlauderer F, et al. Molecular architecture and regulation of BCL10-MALT1 filaments. Nat Commun. 2018;9(1):4041.

45. Baens M, et al. MALT1 auto-proteolysis is essential for NF- $\mathrm{BB}$-dependent gene transcription in activated lymphocytes. PLoS One. 2014;9(8):e103774.

46. Dale LB, et al. G protein-coupled receptor kinase-mediated desensitization of metabotropic glutamate receptor $1 \mathrm{~A}$ protects against cell death. J Biol Chem. 2000;275(49):38213-38220.

47. Ruefli-Brasse AA, French DM, Dixit VM. Regulation of NF- $\mathrm{BB}$-dependent lymphocyte activation and development by paracaspase. Science. 2003;302(5650):1581-1584.

48. Liao W, Lin JX, Leonard WJ. Interleukin-2 at the crossroads of effector responses, tolerance, and immunotherapy. Immunity. 2013;38(1):13-25.

49. Arnon TI, et al. GRK2-dependent S1PR1 desensitization is required for lymphocytes to overcome their attraction to blood. Science. 2011;333(6051):1898-1903.

50. Brinkman EK, Chen T, Amendola M, van Steensel B. Easy quantitative assessment of genome editing by sequence trace decomposition. Nucleic Acids Res. 2014;42(22):e168.

51. Compagno M, et al. Mutations of multiple genes cause deregulation of NF- $\kappa \mathrm{B}$ in diffuse large B-cell lymphoma. Nature. 2009;459(7247):717-721.

52. Visco C, et al. Comprehensive gene expression profiling and immunohistochemical studies support application of immunophenotypic algorithm for molecular subtype classification in diffuse large B-cell lymphoma: a report from the International DLBCL Rituximab-CHOP Consortium Program Study. Leukemia. 2012;26(9):2103-2113.

53. Dave SS, et al. Molecular diagnosis of Burkitt's lymphoma. N Engl J Med. 2006;354(23):2431-2442.

54. Lenz G, et al. Stromal gene signatures in large-B-cell lymphomas. $N$ Engl J Med. 2008;359(22):2313-2323.

55. Lam LT, et al. Cooperative signaling through the signal transducer and activator of transcription 3 and nuclear factor- $\kappa \mathrm{B}$ pathways in subtypes of diffuse large B-cell lymphoma. Blood. 2008;111(7):3701-3713.

56. Nacinović-Duletić A, Stifter S, Dvornik S, Skunca Z, Jonjić N. Correlation of serum IL-6, IL-8 and IL-10 levels with clinicopathological features and prognosis in patients with diffuse large B-cell lymphoma. Int J Lab Hematol. 2008;30(3):230-239.

57. Akagi T, et al. A novel gene, MALT1 at 18q21, is involved in $\mathrm{t}(11 ; 18)$ ( $\mathrm{q} 21 ; \mathrm{q} 21)$ found in low-grade B-cell lymphoma of mucosa-associated lymphoid tissue. Oncogene. 1999;18(42):5785-5794.

58. Dierlamm J, et al. The apoptosis inhibitor gene API2 and a novel 18q gene, MLT, are recurrently rearranged in the $\mathrm{t}(11 ; 18)(\mathrm{q} 21 ; \mathrm{q} 21)$ associated with mucosa-associated lymphoid tissue lymphomas. Blood. 1999;93(11):3601-3609.

59. Morgan JA, et al. Breakpoints of the $t(11 ; 18)$ (q21;q21) in mucosa-associated lymphoid tissue (MALT) lymphoma lie within or near the previously undescribed gene MALT1 in chromosome 18. Cancer Res. 1999;59(24):6205-6213.

60. Suzuki H, Motegi M, Akagi T, Hosokawa Y, Seto M. API1-MALT1-MLT is involved in mucosa-associated lymphoid tissue lymphoma with $\mathrm{t}(11 ; 18)$ (q21;q21). Blood.1999;94(9):3270-3271.

61. Hara $\mathrm{H}$, et al. The MAGUK family protein CARD11 is essential for lymphocyte activation. Immunity. 2003;18(6):763-775.

62. Ruland J, et al. Bcl10 is a positive regulator of antigen receptor-induced activation of NF- $\mathrm{B}$ and neural tube closure. Cell. 2001;104(1):33-42.

63. Kataoka K, et al. Integrated molecular analysis of adult T cell leukemia/lymphoma. Nat Genet. 2015;47(11):1304-1315.

64. Wang L, et al. Genomic profiling of Sézary syndrome identifies alterations of key T cell signaling and differentiation genes. Nat Genet. 2015;47(12):1426-1434.

65. da Silva Almeida AC, et al. The mutational landscape of cutaneous T cell lymphoma and Sézary syndrome. Nat Genet. 2015;47(12):1465-1470.

66. McAllister-Lucas LM, Lucas PC. Finally, MALT1 is a protease! Nat Immunol. 2008;9(3):231-233.

67. Yamasoba D, et al. N4BP1 restricts HIV-1 and its inactivation by MALT1 promotes viral reactivation. Nat Microbiol. 2019;4(9):1532-1544.

68. Knies N, et al. Lymphomagenic CARD11/ BCL10/MALT1 signaling drives malignant B-cell proliferation via cooperative NF- $\kappa \mathrm{B}$ and JNK activation. Proc Natl Acad Sci U S A. 2015;112(52):E7230-E7238.

69. Uehata T, et al. Malt1-induced cleavage of regnase- 1 in $\mathrm{CD} 4(+)$ helper $\mathrm{T}$ cells regulates immune activation. Cell. 2013;153(5):1036-1049.

70. Jeltsch KM, et al. Cleavage of roquin and regnase- 1 by the paracaspase MALT1 releases their cooperatively repressed targets to promote $\mathrm{T}(\mathrm{H}) 17$ differentiation. Nat Immunol. 2014;15(11):1079-1089.

71. Xin BT, et al. Development of new Malt1 inhibitors and probes. Bioorg Med Chem. 2016;24(15):3312-3329.

72. Lim SM, et al. Identification of $\beta$-lapachone analogs as novel MALT1 inhibitors to treat an aggressive subtype of diffuse large B-cell lymphoma. JMed Chem. 2015;58(21):8491-8502.

73. Quancard J, et al. An allosteric MALT1 inhibitor is a molecular corrector rescuing function in an immunodeficient patient. Nat Chem Biol. 2019;15(3):304-313.

74. Watari K, Nakaya M, Kurose H. Multiple functions of $\mathrm{G}$ protein-coupled receptor kinases. J Mol Signal. 2014;9(1):1. 
75. Nogués L, et al. G Protein-coupled receptor kinase 2 (GRK2) promotes breast tumorigenesis through a HDAC6-Pin1 axis. EBioMedicine. 2016;13:132-145.

76. Buchholz M, et al. A multistep high-content screening approach to identify novel functionally relevant target genes in pancreatic cancer. PLoS One. 2015;10(4):e0122946.

77. Zhou L, et al. G-protein-coupled receptor kinase 2 in pancreatic cancer: clinicopathologic and prognostic significance. Hum Pathol. 2016;56:171-177.

78. Wei Z, Hurtt R, Ciccarelli M, Koch WJ, Doria C. Growth inhibition of human hepatocellular carcinoma cells by overexpression of G-proteincoupled receptor kinase 2.J Cell Physiol. 2012;227(6):2371-2377.

79. Wei Z, Hurtt R, Gu T, Bodzin AS, Koch WJ, Doria
C. GRK2 negatively regulates IGF-1R signaling pathway and cyclins' expression in HepG2 cells. JCell Physiol. 2013;228(9):1897-1901.

80. Ribas C, et al. The G protein-coupled receptor kinase (GRK) interactome: role of GRKs in GPCR regulation and signaling. Biochim Biophys Acta. 2007;1768(4):913-922.

81. Nogués L, et al. G protein-coupled receptor kinases (GRKs) in tumorigenesis and cancer progression: GPCR regulators and signaling hubs. Semin Cancer Biol. 2018;48:78-90.

82. Kong G, Penn R, Benovic JL. A $\beta$-adrenergic receptor kinase dominant negative mutant attenuates desensitization of the $\beta 2$-adrenergic receptor. J Biol Chem. 1994;269(18):13084-13087.

83. Levay K, Satpaev DK, Pronin AN, Benovic JL, Slepak VZ. Localization of the sites for $\mathrm{Ca}^{2+}$-bind- ing proteins on $\mathrm{G}$ protein-coupled receptor kinases. Biochemistry. 1998;37(39):13650-13659.

84. Patial S, Luo J, Porter KJ, Benovic JL, Parameswaran N. G-protein-coupled-receptor kinases mediate $\mathrm{TNF} \alpha$-induced $\mathrm{NF} \kappa \mathrm{B}$ signalling via direct interaction with and phosphorylation of IkB $\alpha$. Biochem J. 2009;425(1):169-178.

85. O'Donnell MA, et al. Caspase 8 inhibits programmed necrosis by processing CYLD. Nat Cell Biol. 2011;13(12):1437-1442.

86. Hailfinger S, Pelzer C, Thome M. Detection and measurement of paracaspase MALT1 activity. Methods Mol Biol. 2014;1133:177-188.

87. Pelzer C, Cabalzar K, Wolf A, Gonzalez M, Lenz $\mathrm{G}$, Thome M. The protease activity of the paracaspase MALT1 is controlled by monoubiquitination. Nat Immunol. 2013;14(4):337-345. 